Document downloaded from:

http://hdl.handle.net/10251/65017

This paper must be cited as:

Naud, B.; Novella Rosa, R.; Pastor Enguídanos, JM.; Winklinger, JF. (2015). RANS modelling of a lifted $\mathrm{H} 2 / \mathrm{N} 2$ flame using an unsteady flamelet progress variable approach with presumed PDF. Combustion and Flame. 162(4):893-906. doi:10.1016/j.combustflame.2014.09.014.

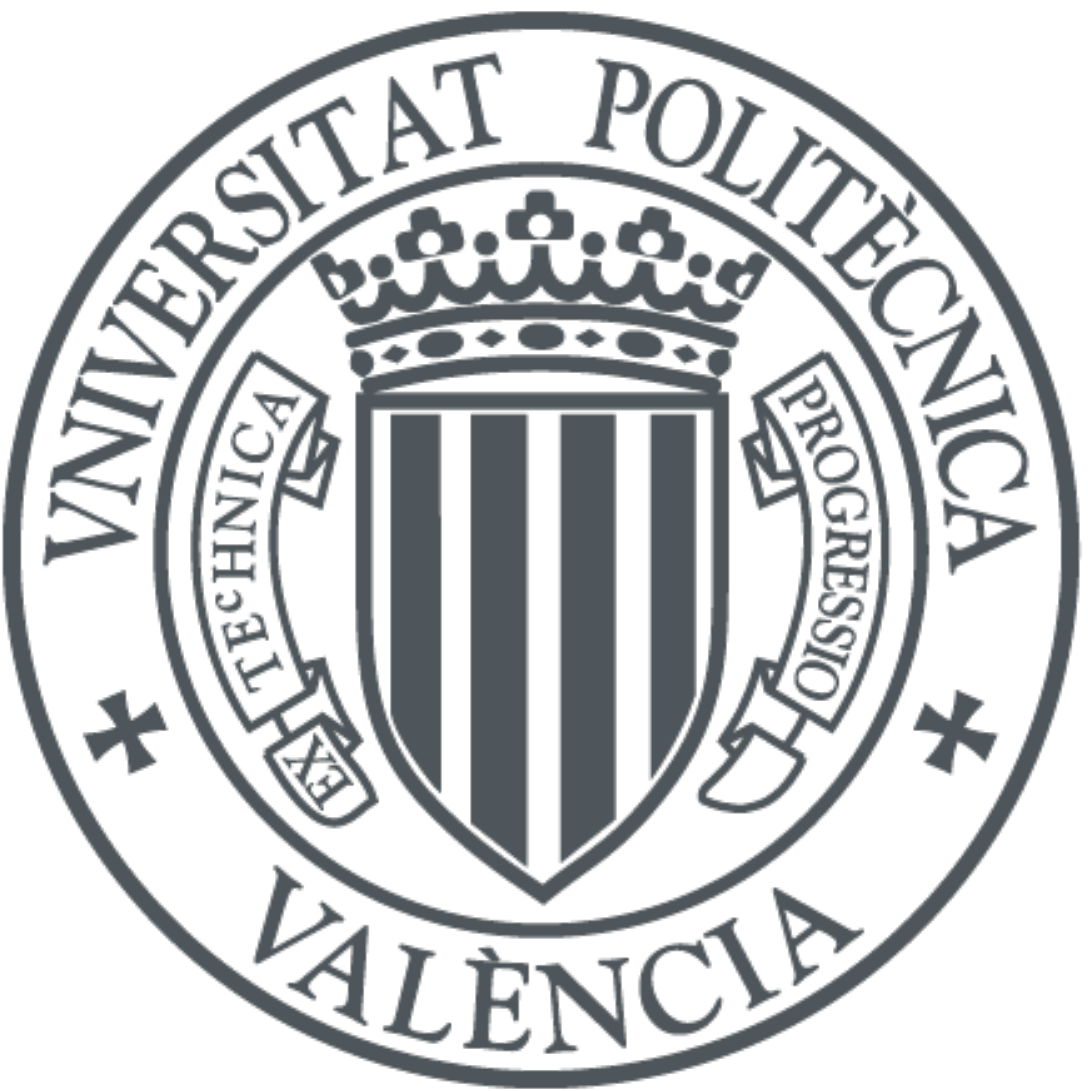

The final publication is available at

http://dx.doi.org/10.1016/j.combustflame.2014.09.014

Copyright Elsevier

Additional Information 


\title{
RANS modelling of a lifted H2/N2 flame using an unsteady flamelet / progress variable approach with presumed PDF
}

\author{
Bertrand Naud $^{\mathrm{a}, *}$, Ricardo Novella ${ }^{\mathrm{b}}$, José Manuel Pastor $^{\mathrm{b}}$, Johannes F. Winklinger ${ }^{\mathrm{b}}$ \\ ${ }^{a}$ Modelling and Numerical Simulation Group, Energy Department, CIEMAT, Avda. Complutense 22, 28040 Madrid, Spain \\ ${ }^{b}$ CMT-Motores Térmicos, Universitat Politècnica de València, Camino de Vera s/n, 46022 Valencia, Spain
}

\begin{abstract}
An unsteady flamelet / progress variable (UFPV) approach is used to model a lifted $\mathrm{H}_{2} / \mathrm{N}_{2}$ flame in a RANS framework together with presumed PDF. We solve the unsteady flamelets both in physical space and in mixture fraction space. We show that in the former case, the scalar dissipation rate profile strongly varies in time (while it is assumed to be fixed in time in the latter). However, this does not result in significant qualitative differences in the corresponding flamelet libraries. The progress variable is carefully defined, including both the main combustion product $\left(\mathrm{H}_{2} \mathrm{O}\right)$ and a key radical species in ignition process $\left(\mathrm{HO}_{2}\right)$. The presumedPDF model is proposed in terms of the non-normalised progress variable, without assuming its statistical independence with mixture fraction. We introduce a modelled transport equation for the mean progress variable which is consistent with the basic underlying UFPV assumption, derived from the Lagrangian flamelet model. The influence of different model parameters on the results for the mean temperature and mean species mass fractions and their fluctuations is discussed. Good results are obtained for the conditions of the considered lifted flame where detailed experimental data is available. However, at low coflow temperature the modelled flame lift-off height is shorter than expected.
\end{abstract}

Keywords: tabulated chemistry manifold, unsteady flamelets, auto-ignition, progress variable, presumed PDF

\section{Introduction}

For the modelling of industrial combustion devices such as diesel engines or gas turbines, the use of tabulated chemistry together with presumed-PDF modelling is an attractive approach. In unsteady flamelet/progress variable (UFPV) models, the strong underlying assumption is to suppose that the structure of the turbulent flame considered can be described by igniting and extinguishing laminar diffusion flamelets [1-3], where the mixture fraction $Z$ and its scalar dissipation rate $\chi$ are key parameters. This kind of approach has received attention lately [4-15], resolving the unsteady flamelet equations in $Z$-space with the assumption of fixed-in-time $\chi$-profile from the analytic steady solution of laminar counterflow diffusion flames.

This paper is a contribution to UFPV modelling with presumed PDF in a RANS framework. In order to deal with realistic conditions while involving a small number of chemical species, we consider a steady lifted $\mathrm{H}_{2} / \mathrm{N}_{2}$ turbulent jet flame in a coflow of hot products at atmospheric pressure (the vitiated co-flow burner developed by Dibble and co-workers), first measured at Berkeley university by Cabra et al. $[16,17]$ and later at Sydney university at different operating conditions $[18,19]$. This flame has mainly been modelled in a RANS framework using transported PDF methods with different mixing models [16, 20-28]. A first CMC calculation of this flame was reported in [29]. First LES calculations were based on stochastic fields

\footnotetext{
${ }^{*}$ Corresponding author

Email address: bertrand.naud@ciemat.es (Bertrand Naud)
}

[30], and on perfectly stirred reactors [31], and more recently LES-CMC calculations were presented [32,33]. In all these different modelling studies, different reduced or complete chemical mechanisms were directly applied. Two main conclusions from the experimental and numerical studies can be drawn: the main stabilisation mechanism of this flame is autoignition (with the key role of $\mathrm{HO}_{2}$ radical on the ignition process), and the liftoff height is strongly sensitive to the coflow temperature.

These modelling approaches are based on a mean scalar dissipation rate (transported PDF) or possibly a conditional mean scalar dissipation rate (CMC). Even if some fluctuations of the scalar dissipation rate are considered in LES models (due to the large scale fluctuations of the gradient of the filtered mixture fraction), none of the above models applied to this flame include the small scale fluctuations of the scalar dissipation rate. These fluctuations are expected to follow some log-normal distribution [34]. We can expect that including such fluctuations would decrease the flame lift-off height, by including contributions of low scalar dissipation rates and therefore decreasing the ignition delay time. However, the general trend of the above-mentioned numerical results is a rather too short flame lift-off height, and the correct flame is obtained at coflow temperatures lower than the nominal experimental one (within the experimental error range). On the other hand, one example of modelling of this lifted $\mathrm{H}_{2} / \mathrm{N}_{2}$ flame using unsteady flamelets is reported by Vicquelin [15]. In this case where $\chi$ fluctuations are accounted for through a log-normal PDF, the correct flame lift-off is obtained by prescribing a slightly higher coflow temperature than the experimental one. This different trend be- 
tween modelling based on direct chemistry integration or based on unsteady flamelets is probably related to the fact that the latter relies on an underlying assumption on the local flame structure, including molecular diffusion effects.

The modelling approach proposed here in a RANS framework is similar to [15] and follows the idea of the unsteady flamelet/progress variable (UFPV) model proposed for LES by Pitsch and Ihme [4], and refined in [6]. The thermochemical properties are parametrised by mixture fraction $Z$, reaction progress parameter $\Lambda$ and stoichiometric scalar dissipation rate $\chi_{\mathrm{st}}$. In $[4,6]$, a presumed PDF model is introduced to represent the filtered density function (FDF) $f_{Z, \Lambda, \chi_{\mathrm{st}}}$, supposing statistical independence between $Z$ and $\Lambda$, and between $Z$ and $\chi_{\text {st }}$, such that: $f_{Z, \Lambda, \chi_{\mathrm{st}}}=f_{Z} \cdot f_{\chi_{\mathrm{st}}} \cdot f_{\Lambda \chi_{\mathrm{st}}}$. A $\beta$-PDF is used for $f_{Z}$ and a $\delta$-PDF for $f_{\chi_{\mathrm{st}}}$. In [4], a $\delta$-PDF is also used for $f_{\Lambda \mid \chi_{\mathrm{st}}}$ while a statistically most likely distribution (SMLD) [5] is employed in [6]. The final look-up table of mean thermochemical properties is expressed directly in terms of the mean mixture fraction $\widetilde{Z}$ and its variance $\widetilde{Z^{\prime \prime 2}}$, the mean scalar dissipation rate $\tilde{\chi}$ (substituting the stoichiometric $\chi_{\text {st }}$ ) and a mean progress variable $\widetilde{Y}_{c}$ and possibly its variance (substituting the reaction progress parameter $\Lambda$ ). A simpler presumed-PDF model was used in LES with only a $\beta$-distribution for mixture fraction in $[7,8]$ and was applied to different methane turbulent flames. The modelling presented in [4] was recently applied to auto-ignition in diesel engine conditions using an unsteady RANS approach in [9] and in [10]. In our case, the presumed-PDF assumptions are rather following the work of Michel et al. [11, 12], also developped in a RANS framework. Using their terminology we use a similiar approach as the "DF-PCM $\chi$ " approach used in [15]. We assume a $\beta$ PDF for $f_{Z}$ and a log-normal distribution for $f_{\chi_{\mathrm{st}}}$. However, we use a $\delta$-PDF for $f_{\Lambda \mid \chi_{\mathrm{st}}}$, with $\Lambda$ the unsteady flamelet Lagrangian time $\tau$, allowing us to consider a non-normalised progress variable dependent on $Z$. This is different from the PCM approach where a $\beta$-PDF is used for the normalised progress variable (in this case a normalised progress variable has to be considered since the $\beta$-distribution requires fixed minimum and maximum values for the considered variable, independent of $Z$ ). We simply express the final look-up table in terms of $\widetilde{Z}, \widetilde{Z^{\prime \prime 2}}, \widetilde{\chi}$ and $\widetilde{Y}_{c}$ as Pitsch and Ihme [4].

In the modelled transport equation for the mean progress variable, Pitsch and Ihme use the mean of the chemical reaction rate $\dot{\omega}_{c}$ as usually done in steady flamelet/progress variable (SFPV) models. On the other hand, when using approximated diffusion flamelets, Michel et al. use a mean source term including both chemical reaction and molecular diffusion: we will denote this term $\dot{Y}_{c}$ (which is zero for steady flamelets where diffusion and reaction counterbalance). The principle of SFPV models is clearly detailed in [35], and such models have been introduced and used by different authors with different names: for instance FGM [36], FPI [37, 38] or REDIM [39]. In SFPV models [35], the progress variable $\boldsymbol{y}_{c}$ - corresponding to the value of mass fractions of combustion products either in steady laminar flames or in the inert mixture (extinguished flames) - is a way to substitute the scalar dissipation rate $\chi$ in the parametrisation of the steady-flamelet models, previously expressed in terms of $(Z, \chi)$. In the SFPV framework, the modelled transport equation of the mean of this 'steady flame combustion product mass fraction', $\boldsymbol{y}_{c}$, is simply written formally as the mean transport equation for a reactive species mass fraction. This modelled equation includes the mean of the reaction term $\dot{\omega}_{c}$ obtained from the steady flamelets, and clipping is applied if necessary. This choice actually implies a shape for the PDF of $\chi$ (which is not formally derived) in such a way that the modelled mean $\chi$ of the numerical solution and the modelling of the PDF of the progress variable $\mathcal{Y}_{c}$ are consistent [35]. When considering UFPV models, a progress variable $Y_{c}$ based on species mass fractions from unsteady flamelets is of different nature of $\mathcal{Y}_{c}$. While formally the same, the transport equation used in [4] is different from the one introduced in [35]. We will discuss here the choice of the modelled transport equation for the mean progress variable, including a formulation in terms of $\dot{\omega}_{c}$, and a formulation in terms of $\dot{Y}_{c}$.

As a final remark, we know that LES potentially allows to model scalar mixing, intermittency, transient effects in a much finer way than RANS. This is especially relevant when considering practical devices which are usually characterised by complex flow patterns and complex geometries. On the other hand, under the high Damköhler number assumption of flamelet-like combustion models, the LES filtered chemical source term needs to be modelled entirely, as in RANS [40]. In the simple steady axisymmetric jet configuration considered, we can expect that our well calibrated RANS simulation can provide enough information in terms of scalar mixing (mean mixture fraction, variance and mean scalar dissipation rate) together with the presumed-PDF assumptions considered $(\beta$-PDF for mixture fraction and log-normal PDF for the stoichiometric scalar dissipation rate). In particular, we expect the assumption of a log-normal PDF for $\chi_{\text {st }}$ to allow to obtain a modelling framework where intermittent ignition events are considered in a good way and where minor species can be as well predicted as compared to LES modelling like [4-6] where a $\delta$-PDF is assumed for $\chi_{\mathrm{st}}$, or compared to LES-CMC like [32, 33]. The proposed combustion model for RANS modelling of simple steady flames could be extended in a straightforward way to LES modelling (with some possible modifications of the presumed-PDF assumptions).

In the next section, the resolution of unsteady flamelets in physical space (where the scalar dissipation rate is not fixed) or in $Z$-space (with a prescribed fixed-in-time $\chi$-profile) is discussed, together with the adequate definition of the progress variable $Y_{c}$. In Section 3 the presumed-PDF assumptions are detailed, considering a non-normalised progress variable. In Section 4, together with the turbulence model, two equations for the mean progress variable are presented: either including the mean of the reaction term $\dot{\omega}_{c}$ or of the flamelet transient source term $\dot{Y}_{c}$. Finally, results are presented together with the influence of different parameters, and some limits of the model are discussed for low coflow temperature where the liftoff height is underpredicted. 


\section{Unsteady flamelet manifolds}

For completeness, we will repeat here the basic flamelet equations and notations. We do not recall here the range of application of flamelet models in turbulent combustion modelling, but we first clearly state what can be a physical interpretation of the model (including the choice of the progress variable for igniting flamelets), which will later have some implications concerning the way to express the presumed-PDF model and the way to write the modelled transport equation for the progress variable.

We then show that the manifold obtained from the transient solutions of unsteady flamelets solved in $Z$-space using the constant steady $\chi$-profile proposed by Peeters is in correspondence with the manifold obtained from unsteady flamelets resolved in physical space, despite the strong unsteadiness of the $\chi$-profile in the later case. Note that, so far, the correspondence derived by Peeters for steady flamelets has been used in the context of unsteady flamelets (solved in $Z$-space) without considering the possible impact of the time-variation of $\chi$.

\subsection{Modelling assumption and 3D parametrization}

The strong underlying assumption in our modelling is to suppose that the structure of the stationary lifted flame considered can be described by igniting and extinguishing laminar flamelets (in the opposed-jet configuration, with the fuel and hot coflow conditions of the considered flame). In this way, at a given point in the turbulent lifted flame, the value of any property $Q(\boldsymbol{x}, t)$ is assumed to correspond to the solution of a given unsteady flamelet defined by the strain rate $a$, after a given time $\Delta \tau_{\text {uns }}$, at a given mixture fraction $Z$. Therefore, we consider all possible solutions of igniting and extinguishing unsteady flamelets in a 3D space parametrised by $Z, a$ and $\Delta \tau_{\text {uns }}$. We refer to the values of any property $Q$ corresponding to the unsteady flamelet solutions as: $Q^{\text {uns }}\left(Z, a, \Delta \tau_{\text {uns }}\right)$.

The underlying assumption of our modelling is then that at any point in the turbulent lifted flame, there exists a given value of $\left(Z, a, \Delta \tau_{\text {uns }}\right)$ (corresponding to the actual local values of the turbulent reacting flow), such that: $Q(\boldsymbol{x}, t)=Q^{\text {uns }}\left(Z, a, \Delta \tau_{\text {uns }}\right)$.

Re-parametrization in terms of local properties. We can indeed measure local values of $Z(\boldsymbol{x}, t)$ in our turbulent flow, using for instance Bilger's formula [41]:

$$
Z=\frac{\left(Y_{\mathrm{H}}-Y_{\mathrm{H}, \mathrm{cof}}\right) / 2 W_{\mathrm{H}}-\left(Y_{\mathrm{O}}-Y_{\mathrm{O}, \mathrm{cof}}\right) / W_{\mathrm{O}}}{\left(Y_{\mathrm{H}, \mathrm{fu}}-Y_{\mathrm{H}, \mathrm{cof}}\right) / 2 W_{\mathrm{H}}-\left(Y_{\mathrm{O}, \mathrm{fu}}-Y_{\mathrm{O}, \mathrm{cof}}\right) / W_{\mathrm{O}}},
$$

where $Y_{\mathrm{H}}$ and $Y_{\mathrm{O}}$ are the elementary mass fractions of $\mathrm{H}$ and $\mathrm{O}$ atoms (and $W_{\mathrm{H}}$ and $W_{\mathrm{O}}$ their respective atomic weight), and where the subscripts 'fu' and 'cof' refer to the fuel and coflow streams as in Table 1.

However, rather than the strain rate $a$ (which defines the boundary conditions of the laminar unsteady flamelet calculation), we rather consider the scalar dissipation rate $\chi$ :

$$
\chi=2 D \frac{\partial Z}{\partial x_{j}} \frac{\partial Z}{\partial x_{j}},
$$

which can be measured as a local value in the turbulent flow (in the above equation $D$ is the diffusion coefficient of mixture fraction).

On the other hand, the definition of the local value of the unsteady flamelet time parameter $\Lambda=\Delta \tau_{\text {uns }}$ is not straightforward. We actually need a parameter giving a physical sense of the time advancement of the unsteady flamelet solution. A good candidate may be a progress variable defined as a linear combination of species mass fractions of combustion products as it has been proposed many times for the construction of tabulated chemistry manifolds based on steady flamelets [36-39]. In the present case, an immediate choice would be to define $Y_{c}$ as the mass fraction of water vapour: $Y_{c}=Y_{\mathrm{H}_{2} \mathrm{O}}$. However, while $Y_{\mathrm{H}_{2} \mathrm{O}}$ should be enough to represent the advancement of reaction after ignition, another species needs to be added in order to represent the first stage of the unsteady flamelet before ignition. Different transported PDF and CMC numerical modelling studies of the flame considered have identified $\mathrm{HO}_{2}$ radical as a key intermediate species [22, 27-30, 32, 33]. We propose to include the mass fraction of $\mathrm{HO}_{2}$ radical in the definition of $Y_{c}$ multiplied by a factor 10: $Y_{c}=Y_{\mathrm{H}_{2} \mathrm{O}}+10 Y_{\mathrm{HO}_{2}}$. This factor 10 was chosen by considering the maximum values of $Y_{\mathrm{H}_{2} \mathrm{O}}$ and $Y_{\mathrm{HO}_{2}}$ around ignition in unsteady igniting flamelets. In this way, $Y_{\mathrm{HO}_{2}}$ is the main contribution to $Y_{c}$ before ignition, it is of the same order as $Y_{\mathrm{H}_{2} \mathrm{O}}$ around ignition and it becomes negligible after ignition where $Y_{\mathrm{H}_{2} \mathrm{O}}$ is the main contribution to $Y_{c}$.

We denote by $z$ the sample space of possible values of $Z(\boldsymbol{x}, t), \psi$ the sample space of possible values of $\chi(\boldsymbol{x}, t)$ and $y_{c}$ the sample-space of possible values of $Y_{c}(\boldsymbol{x}, t)$. The unsteady flamelet properties can be represented in the sample space as $Q^{\star}\left(z, \psi, y_{c}\right)$. Note that we want to define $Y_{c}$ such that there exists a function $\tau^{\star}\left(z, \psi, y_{c}\right)$ relating $Y_{c}$ and $\Delta \tau_{\text {uns }}$ :

$$
\Delta \tau_{\mathrm{uns}}=\tau^{\star}\left[Z(\boldsymbol{x}, t), \chi(\boldsymbol{x}, t), Y_{c}(\boldsymbol{x}, t)\right]
$$

where the function $\tau^{\star}\left(z, \psi, y_{c}\right)$ is monotonic in $y_{c}$ for fixed values of $(z, \psi)$, such that we can write the one-to-one correspondence:

$$
Q^{\star}\left[z, \chi^{\mathrm{uns}}\left(z, a, \tau^{\star}\left(z, \psi, y_{c}\right)\right), y_{c}\right]=Q^{\mathrm{uns}}\left[z, a, \tau^{\star}\left(z, \psi, y_{c}\right)\right],
$$

and the unsteady flamelet properties can indeed be represented in an unequivocal way in the sample space as $Q^{\star}\left(z, \psi, y_{c}\right)$. When solving the unsteady flamelet equations in physical space, both $\chi$ and $Y_{c}$ are function of $\left(Z, a, \Delta \tau_{\text {uns }}\right)$. In the case of the lifted flame considered, in order to ensure the one-to-one correspondence (4), we add some contribution of $\mathrm{H}_{2}$ mass fraction in the definition of $Y_{c}$ :

$$
Y_{c}=Y_{\mathrm{H}_{2} \mathrm{O}}+10 Y_{\mathrm{HO}_{2}}+3 Y_{\mathrm{H}_{2}} \text {. }
$$

\subsection{Numerical resolution of $1 D$ flamelets}

The 1D steady and unsteady solutions on the symmetry axis of the planar opposed-jet configuration problem are obtained by resolving the system of equations expressed either in physical space, Eq. (6)-(10), or in Z-space, Eq. (11), using the same computer code LFLAM, developed at Ciemat. The fuel and 
Table 1: Conditions of temperature and species mole fractions of fuel (fu) and hot coflow (cof), corresponding to the atmospheric lifted $\mathrm{H}_{2}$ turbulent jet flame of [16]. The stoichiometric mixture fraction is $Z_{\mathrm{st}}=0.4789$.

\begin{tabular}{|l|l|l|}
\hline & Fuel $(Z=1)$ & Hot coflow $(Z=0)$ \\
\hline Temperature & $T_{\mathrm{fu}}=305 K$ & $T_{\text {cof }}=1045 K$ \\
\hline Mole fractions & $X_{\mathrm{H}_{2}, \mathrm{fu}}=0.25$ & $X_{\mathrm{O}_{2}, \text { cof }}=0.14744$ \\
& $X_{\mathrm{N}_{2}, \mathrm{fu}}=0.75$ & $X_{\mathrm{N}_{2}, \text { cof }}=0.75363$ \\
& & $X_{\mathrm{H}_{2} \mathrm{O}, \text { cof }}=0.09893$ \\
\hline
\end{tabular}

hot coflow conditions used in this paper are summarised in Table 1 . In this case, the stoichiometric mixture fraction is equal to $Z_{\text {st }}=0.4789$. The chemical mechanism of [42] is used, involving 9 species $\left(\mathrm{H}_{2}, \mathrm{H}, \mathrm{O}_{2}, \mathrm{OH}, \mathrm{O}, \mathrm{H}_{2} \mathrm{O}, \mathrm{HO}_{2}, \mathrm{H}_{2} \mathrm{O}_{2}\right.$ and $\left.\mathrm{N}_{2}\right)$ and 21 reactions.

In physical space, we solve the continuity, momentum, species and temperature equations as described in [43, 44] for the planar geometry, here written in their unsteady form (in this formulation $F=\rho u$ and $G=-\rho v / y$ with $u$ the axial and $v$ the normal velocity components and $y$ the perpendicular direction):

$$
\begin{aligned}
& \frac{\partial \rho}{\partial t}=G-\frac{\partial F}{\partial x}, \\
& \frac{\partial G}{\partial t}=-F \frac{\partial}{\partial x}\left(\frac{G}{\rho}\right)+\frac{\partial}{\partial x}\left[\mu \frac{\partial}{\partial x}\left(\frac{G}{\rho}\right)\right]+H+\frac{G^{2}}{\rho}, \\
& \rho \overline{c_{p}} \frac{\partial T}{\partial t}=-\left[\overline{c_{p}} F+\sum c_{p k}\left(\rho Y_{k} V_{k}\right)\right] \frac{\partial T}{\partial x} \\
& +\frac{\partial}{\partial x}\left[\lambda \frac{\partial T}{\partial x}\right]-\sum h_{k} \dot{\omega}_{k} \\
& \rho \frac{\partial Y_{k}}{\partial t}=-F \frac{\partial Y_{k}}{\partial x}+\frac{\partial}{\partial x}\left[-\rho Y_{k} V_{k}\right]+\rho \dot{\omega}_{k},
\end{aligned}
$$

where the transport and thermodynamic coefficients, $\mu, \lambda, c_{p k}$, $h_{k}$ (resp. dynamic molecular viscosity, thermal conductivity, specific heat capacity of species $k$ and enthalpy of species $k$ ) are obtained from temperature-dependent polynomial fits in Chemkin format. Here, the assumption of unity Lewis number reads:

$$
\rho Y_{k} V_{k}=-\rho D \frac{W_{k}}{\bar{W}} \frac{\partial X_{k}}{\partial x} \quad \text { and } \quad D=\frac{\lambda}{\rho \overline{c_{p}}},
$$

where $W_{k}$ is the molar mass and $X_{k}$ the mole fraction of species $k$, and where $\bar{W}$ is the molar mass and $\overline{c_{p}}$ the specific heat capacity of the mixture. The mixture density $\rho$ is obtained from the ideal gas law at atmospheric pressure. A fixed stagnation point is specified at the middle of the domain in order to discretise the equations, and the boundary conditions are specified according to the potential flow assumption on both sides of the $1 \mathrm{D}$ space $(G=d F / d x=$ constant $)$, with: $G_{\text {cof }}=-a . \rho_{\text {cof }}$, $H=-G_{\text {cof }}^{2} / \rho_{\text {cof }}$ and $G_{\text {fu }}=G_{\text {cof }} \cdot\left(\rho_{\text {fu }} / \rho_{\text {cof }}\right)$. By solving the above equations, we obtain the composition and temperature in physical space, $Y_{k}(x)$ and $T(x)$ [at discrete times when solving the unsteady equations]. From the composition, we can retrieve the mixture fraction $Z(x)$ and the scalar dissipation $\chi(x)$, using (1) and (2). We can then express our results in $Z$-space: $T(Z)$, $Y_{k}(Z), \chi(Z)$.

When solving the equations in $Z$-space, we simply solve the following equations for the mass fraction of each species $k$ :

$$
\frac{\partial Y_{k}}{\partial t}=\frac{\chi}{2} \frac{\partial^{2} Y_{k}}{\partial Z^{2}}+\dot{\omega}_{k}
$$

where the scalar dissipation rate profile is prescribed (and fixed in time) in $Z$-space as the steady-state profile from the analytic solution of laminar planar counterflow diffusion flames [1, 2]. This profile, derived by Peters, corresponds to $\chi^{\mathrm{uns}}\left(Z, a, \Delta \tau_{\mathrm{uns}}\right)$ for the steady flamelet solution obtained after a long enough time $\left(\Delta \tau_{\text {uns }}=\infty\right)$ :

$$
\chi^{\infty}(a, Z)=\frac{a}{\pi} \exp \left[-2\left(\operatorname{erfc}^{-1}(2 Z)\right)^{2}\right]
$$

with $\operatorname{erfc}(x)=1-\operatorname{erf}(x)=(2 / \sqrt{\pi}) \int_{x}^{\infty} \exp \left(-y^{2}\right) \cdot d y$. With the assumption of unity Lewis number, we approximate the total enthalpy of the mixture as $h=Z \cdot h_{\mathrm{fu}}+(1-Z) \cdot h_{\text {cof }}$. The temperature is simply obtained as function of composition $Y_{k}$ and total enthalpy $h$.

The reaction rate of species $k$ - last term in (9) and (11) is obtained from the chemical mechanism of [42] in Chemkin format, as function of composition $Y_{k}$ and temperature $T$. In this section, the system of equations (6)-(10) and the system of equations (11)-(12) are solved in their steady or unsteady forms using the algorithm "Twopnt program for boundary value problems" presented in [45]. The final unsteady resolution of (11)-(12) is performed using the DDASSL solver [46], using the exact block tridiagonal Jacobian matrix.

Note that Equation (12) can be written in a different way by introducing the stoichiometric mixture fraction $Z_{\mathrm{st}}$, and the stoichiometric scalar dissipation rate $\chi_{\mathrm{st}}=\chi^{\infty}\left(a, Z_{\mathrm{st}}\right)$ :

$$
\chi^{\infty}\left(\chi_{\mathrm{st}}, Z\right)=\chi_{\mathrm{st}} \mathcal{F}_{\mathrm{st}}^{\infty}(Z),
$$

where $\mathcal{F}_{\text {st }}^{\infty}(Z)=\chi^{\infty}(a, Z) / \chi^{\infty}\left(a, Z_{\text {st }}\right)$ is independent of the strain rate $a$.

\subsection{Flamelets solved in physical space or in Z-space}

Steady solutions: S-curve. Before considering filling in the 3D-space $\left(Z, \chi, Y_{c}\right)$ with all possible igniting and extinguishing unsteady flamelets, we first need to obtain the steady solutions which define the physical limits of ignition and extinction. Figures 1 and 2 show the results for the two types of flamelet solutions. The so-called S-curve is represented in the 3D-space. The zone below the stable branches (red surface and black surface) and above the unstable branch (blue surface) corresponds to the zone of autoignition. Elsewhere (above the black surface and below the blue surface) extinction occurs.

Unsteady solutions: filling in the $\left(Z, \chi, Y_{c}\right)$-space. We can now consider the full 3D manifolds obtained from unsteady igniting and extinguishing flamelets. At low strain rates, we start from the inert solution and let the solution ignite. At higher strain 


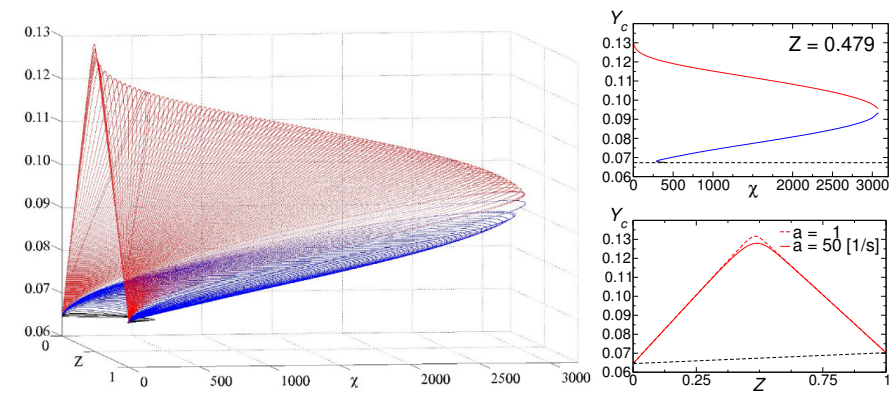

Figure 1: Steady flamelets solved in physical space from Eq. (6)-(10). Left: representation in $\left(Z, \chi, Y_{c}\right)$-space, in red the upper stable branch, in blue the middle unstable branch and in black the lower stable branch. Top right: cut at $Z=0.479$, including inert solution (dashed black line). Bottom right: steady (red) and inert (dashed black) flamelets at strain rates $a=1 \mathrm{~s}^{-1}$ and $a=50 \mathrm{~s}^{-1}$.

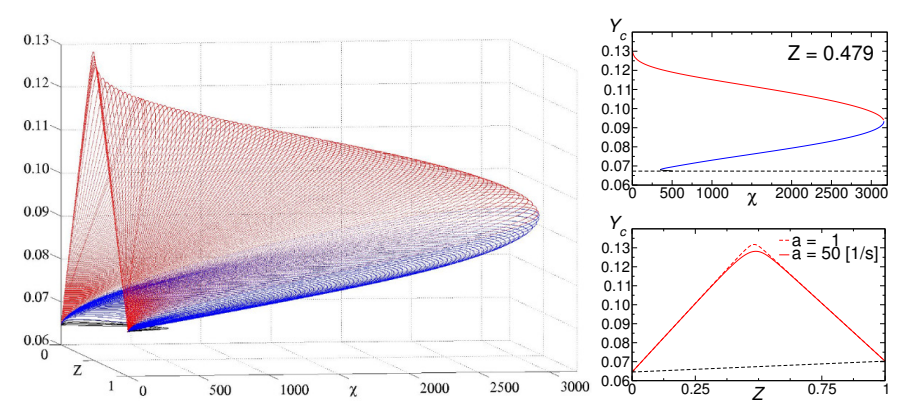

Figure 2: Steady flamelets solved in Z-space from Eq. (11) and (12). See legend in Fig. 1.

rates where different steady solutions exist (stable and unstable), starting from the solution on the unstable middle branch, we slightly decrease the strain rate and let the solution ignite, or we slightly increase the strain rate and let the solution extinguish.

A major difference between unsteady flamelet solutions resolved in physical space or in $Z$-space is that in the first case the scalar dissipation rate varies in time. As an example, Figures 3 and 4 show the time evolution of $\chi(Z)$ for the unsteady igniting flamelet at strain rate $a=50 \mathrm{~s}^{-1}$. We see that, when resolving in physical space, the initial inert $\chi$-profile is lower than the final steady flame $\chi$-profile, and that the fixed-in-time $\chi$-profile used to resolve in $Z$-space lies in between. The main observation here is the strong variation in time of $\chi(Z)$ when resolving in physical space: a strong increase is observed where the mixture ignites, reaching a maximum and then decreasing to the steady flame value. As a consequence, in Figure 5, we observe the wider profiles of $Y_{c}(Z)$ when resolving in physical space due to local higher $\chi$ values (implying larger molecular diffusion effects).

In Figure 6, we plot the unsteady igniting and extinguishing flamelet solutions in given $Z$-slices of the $\left(Z, \chi, Y_{c}\right)$ domain represented in Figure 1 and 2. Obviously for the solutions resolved in $Z$-space, the trajectories of all unsteady flamelets are vertical lines since $\chi(Z)$ is constant in time. However, when resolved in physical space, we observe that for igniting flamelets (upwards trajectories between the middle branch and the upper branch), the scalar dissipation rate first increases and then de-

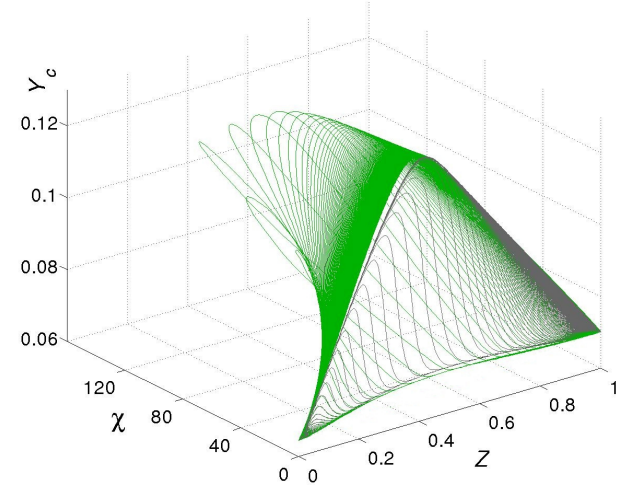

Figure 3: Time evolution (every $10^{-4} \mathrm{~s}$ ) of the unsteady igniting flamelet at strain rate $a=50 \mathrm{~s}^{-1}$. Dark grey: flamelet resolved in $Z$-space (fixed-in-time $\chi(Z)$ profile). Green: flamelet resolved in physical space.

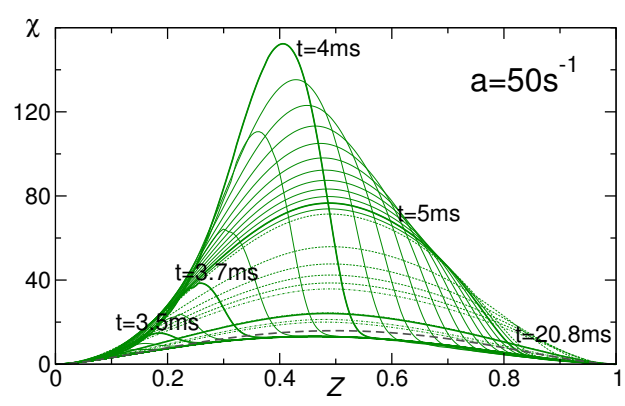

Figure 4: Projection of Fig. 3 on $(Z, \chi)$. Dashed grey line: $\chi(Z)$ profile from Eq. (12). Green lines: time evolution of the igniting flamelet resolved in physical space $\left(10^{-4} \mathrm{~s}\right.$ between the solid lines, $10^{-3} \mathrm{~s}$ between the dashed lines, $10^{-2} \mathrm{~s}$ between the dotted-dashed lines). The thick lines show the solutions at indicated discrete times.

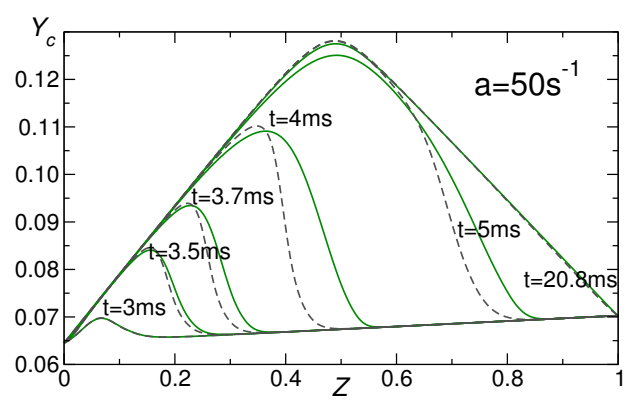

Figure 5: Projection of Fig. 3 on $\left(Z, Y_{c}\right)$ at the discrete times indicated in Fig. 4. Dashed grey lines: solutions of Eq. (11). Green lines: solutions of Eq. (6)-(10).

creases (as observed in Figure 3 and 4), and for extinguishing flamelets (downwards trajectories below the middle branch), $\chi$ first decreases and then increases. As a consequence, we also observe that the $3 \mathrm{D}\left(Z, \chi, Y_{c}\right)$ domains are not filled in in the same way when resolving in physical space or in $Z$-space: the latter allowing to fill in more of the 3D domain.

Flamelet manifolds in $(Z, \chi, Y c)$-space. We make a qualitative comparison of the final manifolds in $\left(Z, \chi, Y_{c}\right)$-space by colouring the trajectories by $\dot{\omega}_{c}$, the reaction rate of the progress variable $Y_{c}$. Since this property is evaluated from the full composition and temperature, it gives a cumulative information of all 
the properties to be possibly stored in the manifold. Moreover, in a later use of the manifold, this property would give important information on how to move within the 3D $\left(Z, \chi, Y_{c}\right)$-space. The general qualitative observation from Figure 6 is that both manifolds are very similar. Although a more detailed quantitative study could be made in the future (in particular also considering the contours of $\Delta \tau_{\text {uns }}$ ), at this stage we can guess that for turbulent combustion modelling, using manifolds based on flamelets resolved in physical space or in $Z$-space would not imply large differences in the results. In the following we will only consider flamelets solved in $Z$-space, with all the simplifications implied by the use of fixed-in-time $\chi$-profiles.
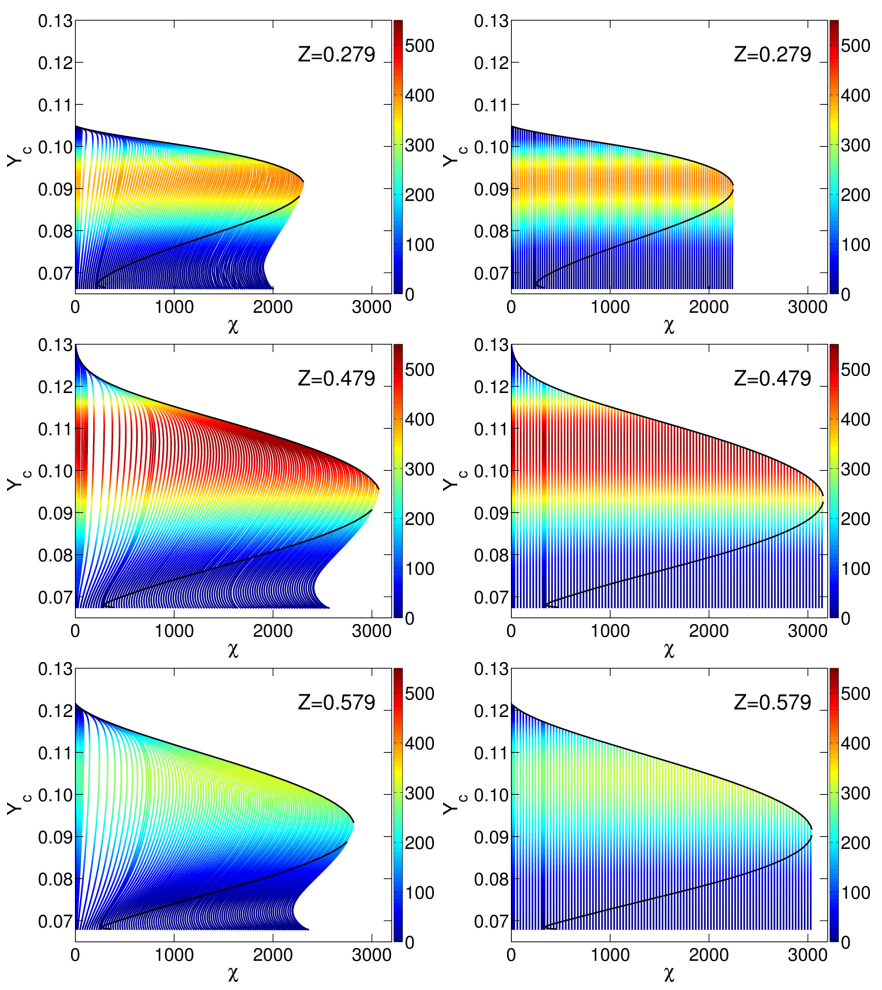

Figure 6: Igniting and extinguishing flamelets coloured by $Y_{c}$ reaction rate, $\dot{\omega}_{c}$. Left: resolved in physical space. Right: resolved in $Z$-space.

\subsection{A priori test of the UFPV model}

At this point, it is interesting to consider the a priori study done by Ihme and See in [6], by considering a Lagrangian Flamelet Model (LFM) [3]. From a RANS calculation of the considered lifted flame, we extract the stoichiometric isosurface from the mean mixture fraction field, where we evaluate $\chi_{\text {st }}$ from the mean scalar dissipation rate. Based on the axial distance from the injector and the mean axial velocity on the stoichiometric iso-surface, we can define a Lagrangian time $\tau_{L}$. As shown in Figure 7(a), we then have a time dependent $\chi_{\mathrm{st}}\left(\tau_{L}\right)$ which can also be represented in function of an adimensional axial distance to the injector exit. We can then solve the LFM equation for the species mass fractions, i.e. Equation (11) with $\chi\left(Z, \tau_{L}\right)=\chi_{\mathrm{st}}\left(\tau_{L}\right) \mathcal{F}_{\mathrm{st}}^{\infty}(Z)$ given by Equation (13). The corresponding time evolution of $Y_{c}$ at $Z_{\mathrm{st}}$ is represented in Figure 7(b). We can also directly solve the UFPV approximation for
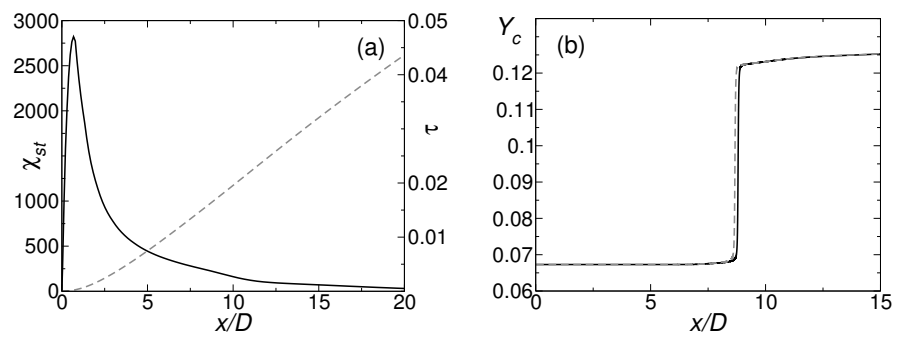

Figure 7: (a) Stoichiometric scalar dissipation rate $\chi_{\text {st }}$ and Lagrangian time $\tau_{L}$ extracted for a RANS calculation of the lifted $\mathrm{H}_{2} / \mathrm{N}_{2}$ flame as functions of an adimensional axial distance. (b) Comparison of the time evolution of $Y_{c}$ at $Z_{\mathrm{st}}$ resolved with LFM (black line) or with the UFPV approximation (dotted grey line).

$Y_{c}\left(Z, \tau_{L}\right)$ instead of (11):

$$
\dot{Y}_{c}=\frac{\partial Y_{c}}{\partial \tau_{L}}=\frac{\chi\left(Z, \tau_{L}\right)}{2} \frac{\partial^{2} Y_{c}}{\partial Z^{2}}+\dot{\omega}_{c}\left[Z, \chi\left(Z, \tau_{L}\right), Y_{c}\left(Z, \tau_{L}\right)\right]
$$

where the reaction term is obtained from the UFPV manifold resolved in $Z$-space, as represented on the right hand side of Figure 6. We see in Figure 7(b) that the UFPV approximation is in very good correspondence with the LFM results. These a priori results for the considered $\mathrm{H}_{2} / \mathrm{N}_{2}$ lifted flame are consistent with the a priori results of Ihme and See for the litfed methane flame [6].

\section{Presumed PDF modelling}

In order to keep the modelling as simple as possible, we want to tabulate the mean properties from the flamelets (mean composition, mean temperature, mean density) as functions of only four parameters: the mean mixture fraction $\widetilde{Z}$, its variance $\widetilde{Z^{\prime \prime 2}}$, the mean scalar dissipation rate $\widetilde{\chi}$ and the mean progress variable $\widetilde{Y}_{c}$. Using the simplifications implied by the resolution of the flamelets in $Z$-space (as usually done in other UFPV approaches), we choose to consider a log-normal distribution for $f_{\chi_{\text {st }}}$ (different from [4-6]) while a non-normalised progress variable is used (different from [11-13, 15]). We do not assume statistical independence between the progress variable and mixture fraction as often considered in presumed PDF modelling (unless their correlation is included as done in [47, 48] using a copula). Instead, we make an assumption of statistical independence using the time evolution of the unsteady flamelet, allowing us to keep a physical dependence between $Y_{c}$ and $Z$.

\subsection{Joint PDF considered}

We have two possible parametrizations of the properties, as expressed in (4). The mean properties can be obtained in two ways:

$$
\begin{aligned}
\widetilde{Q}(\boldsymbol{x}, t)= & \iiint Q^{\star}\left(z, \psi, y_{c}\right) \cdot f_{Z, \chi, Y_{c}}\left(z, \psi, y_{c} ; \boldsymbol{x}, t\right) \cdot \mathrm{d} z \cdot \mathrm{d} \psi \cdot \mathrm{d} y_{c} \\
& =\iiint Q^{\mathrm{uns}}(z, \alpha, \tau) \cdot f_{Z, a, \tau^{\star}}(z, \alpha, \tau ; \boldsymbol{x}, t) \cdot \mathrm{d} z \cdot \mathrm{d} \alpha \cdot \mathrm{d} \tau,
\end{aligned}
$$

where the joint PDFs are introduced in the sense of Favre PDFs. The first representation is not a good starting point for 
presumed-PDF modelling since $Z, \chi$ and $Y_{c}$ are all interdependent [49]. In order to obtain a tabulation in terms of $\widetilde{Z}, \widetilde{Z^{\prime \prime 2}}$, $\widetilde{\chi}$ and $\widetilde{Y}_{c}$, we rather start from the second representation where we can reasonably assume statistical independence of the variables $Z$ and $a$ on the one hand, and $Z$ and $\tau^{\star}$ on the other hand. Still, in principle $\tau^{\star}$ is dependent on $a$ since the time evolution of the unsteady flamelet is obviously strongly related to the strain rate. We can then write the joint PDF $f_{Z, a, \tau^{\star}}$ as the product of the marginal Z-PDF and the joint $\left(a, \tau^{\star}\right)$-PDF: $f_{Z, a, \tau^{\star}}=f_{Z} \cdot f_{a, \tau^{\star}}=f_{Z} \cdot f_{\tau^{\star} \mid a} \cdot f_{a}$.

We solve the flamelets in $Z$-space, so the $\chi$-profile given in Eq. (12) as a function of $a$ can also be expressed as a function of $\chi_{\mathrm{st}},(13)$, since $\chi_{\mathrm{st}}$ and $a$ are simply related by a constant multiplying factor. As usually done in the literature, in the following we rather consider $\chi_{\text {st }}$ instead of $a$, and we consider the joint PDF:

$$
f_{Z, \chi_{\mathrm{st}}, \tau^{\star}}\left(z, \psi_{\mathrm{st}}, \tau\right)=f_{Z}(z) \cdot f_{\tau^{\star}} \chi_{\mathrm{st}}\left(\psi_{\mathrm{st}}, \tau\right) \cdot f_{\chi_{\mathrm{st}}}\left(\psi_{\mathrm{st}}\right),
$$

and the flamelet properties $Q^{\mathrm{uns}}\left(z, \psi_{\mathrm{st}}, \tau\right)$.

We now detail how to obtain the mean properties as functions of $\widetilde{Z}, \widetilde{Z^{\prime \prime 2}}, \widetilde{\chi}$ and $\widetilde{Y}_{c}$, by assuming a $\beta$-PDF for $f_{Z}$, a $\delta$-PDF for $f_{\tau^{\star} \mid \chi_{\mathrm{st}}}$ and a log-normal for $f_{\chi_{\mathrm{st}}}$.

\subsection{Conditional mean properties $\left\langle Q \mid \chi_{\mathrm{st}}, \tau^{\star}\right\rangle$}

We make the usual assumption that the PDF of $Z$ can be modelled as a $\beta$-distribution based on the unconditional mean value $\widetilde{Z}(\boldsymbol{x}, t)$ and its variance $\widetilde{Z^{\prime \prime 2}}(\boldsymbol{x}, t): f_{Z}(z ; \boldsymbol{x}, t)=f_{\widetilde{Z}, \widetilde{Z^{\prime \prime 2}}}^{\beta}(z ; \boldsymbol{x}, t)$. Вy integration in $Z$-space we obtain the conditional means:

$$
\left\langle Q \mid \chi_{\mathrm{st}}, \tau^{\star}\right\rangle\left(\widetilde{Z}, \widetilde{Z^{\prime \prime 2}}, \psi_{\mathrm{st}}, \tau\right)=\int_{[Z]} Q^{\mathrm{uns}}\left(z, \psi_{\mathrm{st}}, \tau\right) \cdot f_{\widetilde{Z}, \widetilde{Z^{\prime \prime 2}}}^{\beta}(z) . \mathrm{d} z .
$$

\subsection{Conditional mean properties $\left\langle Q \mid \chi_{\mathrm{st}}\right\rangle$}

At this point, we want to model the conditional PDF $f_{\tau^{\star}} \chi_{\mathrm{st}}\left(\psi_{\mathrm{st}}, \tau\right)$ and integrate over $\tau^{\star}$ in order to obtain the conditional means $\left\langle Q \mid \chi_{\text {st }}\right\rangle$. The simplest assumption is to suppose a $\delta$-PDF for $f_{\tau^{\star} \mid \chi_{\mathrm{st}}}$. In other terms, we assume that there are no fluctuations of $\tau^{\star}$ for a given value of $\chi_{\mathrm{st}}$, and the conditional expected value $\left\langle\tau^{\star} \mid \chi_{\text {st }}\right\rangle$ has to be specified. With this assumption, in the final model all the fluctuations are due to fluctuations of mixture fraction and its scalar dissipation rate.

This assumption is made in order to simplify the modelling and in order to lead to an easier reparametrization in terms of the mean progress variable $\widetilde{Y}_{c}$, while keeping the physical sense of the dependence of $Y_{c}$ on $Z$ in igniting flamelets. It can be better understood by looking at Figure 5: given the value of $\chi_{\text {st }}$ or $a$ (which fluctuates in the turbulent flame) we consider the profiles as function of $Z$ for given values of $\tau^{\star}$, without considering fluctuations of $\tau^{\star}$ conditional on $a$. By definition of the progress variable, for given values of $z$ and $\psi_{\text {st }}, Y_{c}^{\text {uns }}$ is a monotonic function of $\tau$ in order to ensure the one-to-one correspondence (4). We can then easily see that $\left\langle Y_{c} \mid \chi_{\text {st }}\right\rangle$ also has a bijective relation with $\left\langle\tau^{\star} \mid \chi_{\mathrm{st}}\right\rangle$ for given values of $\widetilde{Z}, \widetilde{Z^{\prime \prime 2}}$ and $\psi_{\text {st }}$. Assuming a $\delta$-PDF for $f_{\tau^{\star} \mid \chi_{\mathrm{st}}}$, the conditional averages can be represented as functions

$$
\left\langle Q \mid \chi_{\mathrm{st}}\right\rangle\left(\widetilde{Z}, \widetilde{Z^{\prime \prime 2}}, \psi_{\mathrm{st}}, \hat{y_{c}}\right)=\left\langle Q \mid \chi_{\mathrm{st}}, \tau^{\star}\right\rangle\left(\widetilde{Z}, \widetilde{Z^{\prime \prime 2}}, \psi_{\mathrm{st}},\left\langle\tau^{\star} \mid \chi_{\mathrm{st}}\right\rangle\right),
$$

where $\hat{y_{c}}$ is the sample space of possible values of $\left\langle Y_{c} \mid \chi_{\text {st }}\right\rangle$. The value of $\left\langle\tau^{\star} \mid \chi_{\text {st }}\right\rangle$ is specified indirectly, by making the assumption that $\left\langle Y_{c} \mid \chi_{\text {st }}\right\rangle$ satisfies the relation:

$$
\hat{y_{c}}=\left\langle Y_{c} \mid \chi_{\mathrm{st}}\right\rangle\left(\widetilde{Z}, \widetilde{Z^{\prime \prime 2}}, \psi_{\mathrm{st}}, \hat{y_{c}}\right)
$$

In the practical implementation of the model, the $\beta$-PDF integration is performed "on-the-fly" for all unsteady flamelets at different $\chi_{\text {st }}$, and intermediate tables of conditional properties $\left\langle Q \mid \chi_{\text {st }}\right\rangle$ are simply stored for every computed $\chi_{\text {st }}$ in a discretised $\left(\widetilde{Z}, \widetilde{Z^{\prime \prime 2}}, \hat{y_{c}}\right)$-space. This can also be better understood by looking at Figure 5. In other terms, the dependence of $Y_{c}$ on $Z$ is included in the model in the form of the profiles of $Y_{c}(Z)$ at given times of advancement of the unsteady ingniting flamelet solution for a given strain rate $a$ (for instance $a=50 s^{-1}$ in Figure 5).

\subsection{Non-normalised progress variable}

Before considering the final $\chi_{\mathrm{st}}-\mathrm{PDF}$ integration, we want to point out that our modelling is based on the non-normalised progress variable. This is different from what has been proposed by others [11-13, 15] where a normalised variable was introduced. This was imposed by their presumed-PDF modelling where they considered a $\beta$-distribution for the progress variable, which required a variable with constant minimum and maximum values.
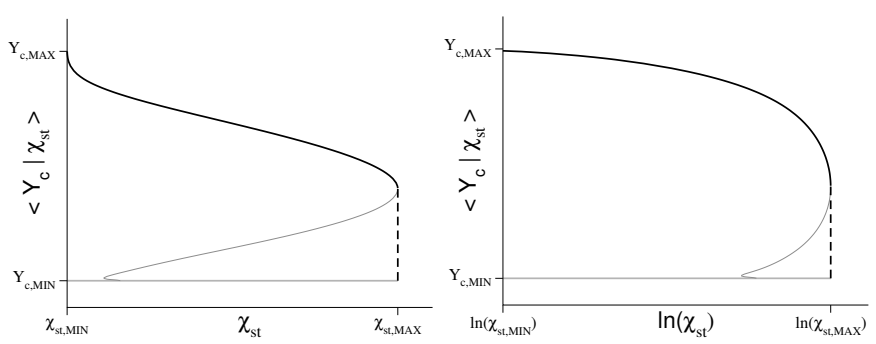

Figure 8: Sketch of the domain of possible values $\left(\psi_{\mathrm{st}}, \hat{y_{c}}\right)$ of $\chi_{\mathrm{st}}$ and $\left\langle Y_{c} \mid \chi_{\mathrm{st}}\right\rangle$ for given values of $\widetilde{Z}$ and $\widetilde{Z^{\prime \prime 2}}$ (represented in logarithmic scale on the right hand side). The bottom grey line represents the inert solution $Y_{c, \mathrm{MIN}}$. The top black line represents the steady state solution $\left\langle Y_{c}^{\max } \mid \chi_{\mathrm{st}}\right\rangle\left(\psi_{\mathrm{st}}\right)$, and together with the dashed black line on the right hand side it represents the limit $\chi_{\mathrm{st}}^{\max }\left(\hat{y_{c}}\right)$.

In Figure 8, we make a sketch of the domain of possible values $\left(\psi_{\mathrm{st}}, \hat{y}_{c}\right)$ of $\chi_{\mathrm{st}}$ and $\left\langle Y_{c} \mid \chi_{\mathrm{st}}\right\rangle$ for given values of $\widetilde{Z}$ and $\widetilde{Z^{\prime \prime 2}}$. This figure is useful in order to make the following observations. At the different strain rates considered (i.e. different given $\left.\chi_{\text {st }}\right)$, the values of $\left\langle Y_{c} \mid \chi_{\mathrm{st}}\right\rangle$ are in between the inert solution value $\left\langle Y_{c}^{\min } \mid \chi_{\mathrm{st}}\right\rangle$ and the steady solution value $\left\langle Y_{c}^{\max } \mid \chi_{\mathrm{st}}\right\rangle$ :

$$
\begin{aligned}
& \left\langle Y_{c}^{\min } \mid \chi_{\mathrm{st}}\right\rangle\left(\widetilde{Z}, \widetilde{Z^{\prime \prime 2}}, \psi_{\mathrm{st}}\right)=\int_{[Z]} Y_{c}^{\min }\left(z, \psi_{\mathrm{st}}\right) \cdot f_{\widetilde{Z}, \widetilde{Z^{\prime \prime 2}}}^{\beta}(z) \cdot \mathrm{d} z, \\
& \left\langle\left. Y_{c}^{\max }\right|_{\mathrm{st}}\right\rangle\left(\widetilde{Z}, \widetilde{Z^{\prime \prime 2}}, \psi_{\mathrm{st}}\right)=\int_{[Z]} Y_{c}^{\max }\left(z, \psi_{\mathrm{st}}\right) \cdot f_{\widetilde{Z}, \widetilde{Z^{\prime \prime 2}}}^{\beta}(z) \cdot \mathrm{d} z .
\end{aligned}
$$

The minimum value is the same inert value for all strain rates:

$$
Y_{c, \mathrm{MIN}}\left(\widetilde{Z}, \widetilde{Z^{\prime \prime 2}}\right)=\left\langle Y_{c}^{\min } \mid \chi_{\mathrm{st}}\right\rangle\left(\widetilde{Z}, \widetilde{Z^{\prime \prime 2}}, \psi_{\mathrm{st}}\right) \quad \text { for all } \psi_{\mathrm{st}}
$$


and there is a maximum $Y_{c, \operatorname{MAX}}\left(\widetilde{Z}, \widetilde{Z^{\prime \prime 2}}\right)$ at the lowest strain rate considered (at $\left.\psi_{\mathrm{st}}=\chi_{\mathrm{st}, \mathrm{MIN}}\right)$ :

$$
Y_{c, \operatorname{MAX}}\left(\widetilde{Z}, \widetilde{Z^{\prime \prime 2}}\right)=\left\langle Y_{c}^{\max } \mid \chi_{\mathrm{st}}\right\rangle\left(\widetilde{Z}, \widetilde{Z^{\prime \prime 2}}, \chi_{\mathrm{st}, \mathrm{MIN}}\right) .
$$

The other way around, for any possible value $\hat{y}_{c}$ of $\left\langle Y_{c} \mid \chi_{\mathrm{st}}\right\rangle$ between $Y_{c, \mathrm{MIN}}$ and $Y_{c, \mathrm{MAX}}$, there is a maximum value $\psi_{\mathrm{st}}=$ $\chi_{\mathrm{st}}^{\max }\left(\widetilde{Z}, \widetilde{Z^{\prime \prime 2}}, \hat{y_{c}}\right)$, corresponding to the steady flamelet solution together with the extinction limit $\chi_{\text {st,MAX }}$.

\subsection{Unconditional mean properties}

The unconditional mean properties are finally obtained from the $\chi_{\mathrm{st}}-\mathrm{PDF}$ integration. As considered by many authors, like in [11-13, 15], we assume a log-normal distribution for $f_{\chi_{\mathrm{st}}}\left(\psi_{\mathrm{st}}\right)=$ $f_{\widetilde{\chi}_{\mathrm{st}}}^{\log }\left(\psi_{\mathrm{st}}\right)$ : we suppose that the PDF of the natural logarithm of $\chi_{\text {st }}$ is a Gaussian distribution with mean $\mu$ and a given fixed variance $\sigma^{2}$, with $\mu=\ln \left(\widetilde{\chi}_{\mathrm{st}}\right)-\sigma^{2} / 2$. For given values of $\widetilde{Z}, \widetilde{Z^{\prime \prime 2}}$ and $\hat{y_{c}}$ (with $Y_{c \text {,MIN }}<\hat{y_{c}}<Y_{c, \mathrm{MAX}}$ ), the unconditional mean properties can be obtained as:

$$
\begin{aligned}
\widetilde{Q}\left(\widetilde{Z}, \widetilde{Z^{\prime \prime 2}}, \widetilde{\chi}_{\mathrm{st}}, \hat{y_{c}}\right) & =\int_{0}^{\chi_{\mathrm{st}}^{\max }}\left\langle Q \mid \chi_{\mathrm{st}}\right\rangle\left(\widetilde{Z}, \widetilde{Z^{\prime \prime 2}}, \psi_{\mathrm{st}}, \hat{y}_{c}\right) \cdot f_{\widetilde{\chi}_{\mathrm{st}}}^{\log }\left(\psi_{\mathrm{st}}\right) \cdot \mathrm{d} \psi_{\mathrm{st}} \\
& +\int_{\chi_{\mathrm{st}}^{\max }}^{\infty} Q_{\chi_{\mathrm{st}}}\left(\widetilde{Z}, \widetilde{Z^{\prime \prime 2}}, \psi_{\mathrm{st}}\right) \cdot f_{\widetilde{\chi}_{\mathrm{st}}}^{\log }\left(\psi_{\mathrm{st}}\right) \cdot \mathrm{d} \psi_{\mathrm{st}} \cdot(24)
\end{aligned}
$$

We had to introduce the notation $Q_{\mid \chi_{\mathrm{st}}}$ for $\psi_{\mathrm{st}}>\chi_{\mathrm{st}}^{\max }\left(\widetilde{Z}, \widetilde{Z^{\prime \prime 2}}, \hat{y_{c}}\right)$ where the conditional averages $\left\langle Q \mid \chi_{\mathrm{st}}\right\rangle$ are not defined (above and on the right hand side of the solid and dashed black lines in Figure 8). We choose to give the steady flamelet property values when $\psi_{\mathrm{st}}>\chi_{\mathrm{st}}^{\max }$ and $\psi_{\mathrm{st}} \leq \chi_{\mathrm{st}, \mathrm{MAX}}$ :

$$
Q_{\chi_{\mathrm{st}}}\left(\widetilde{Z}, \widetilde{Z^{\prime \prime 2}}, \psi_{\mathrm{st}}\right)=\left\langle Q \mid \chi_{\mathrm{st}}\right\rangle\left(\widetilde{Z}, \widetilde{Z^{\prime \prime 2}}, \psi_{\mathrm{st}},\left\langle\left. Y_{c}^{\mathrm{max}}\right|_{\chi_{\mathrm{st}}}\right\rangle\right),
$$

and the inert mixing property values above the extinction limit when $\psi_{\text {st }}>\chi_{\text {st,MAX: }}$

$$
Q_{\mid \chi_{\mathrm{st}}}\left(\widetilde{Z}, \widetilde{Z^{\prime \prime 2}}, \psi_{\mathrm{st}}\right)=\left\langle Q \mid \chi_{\mathrm{st}}\right\rangle\left(\widetilde{Z}, \widetilde{Z^{\prime \prime 2}}, \chi_{\mathrm{st}, \mathrm{MAX}}, Y_{c, \mathrm{MIN}}\right) .
$$

Considering the modelling of the unconditional mean properties in (24), we want to stress once more the fact that the conditional mean properties $\left\langle Q \mid \chi_{\mathrm{st}}\right\rangle$ are expressed in terms of the non-normalised progress variable $\left\langle Y_{c} \mid \chi_{\text {st }}\right\rangle$ (in the $\hat{y_{c}}$-sample space). This tells us how the mean property values include the $\chi_{\mathrm{st}}-$ fluctuations in this model: by averaging over a given value $\hat{y_{c}}$ of $\left\langle Y_{c} \mid \chi_{\mathrm{st}}\right\rangle$. We believe that it is necessary to consider a non-normalised progress variable in the present case where the definition of the progress variable in Eq. (5) takes into account the different stages of autoignition by including $Y_{\mathrm{HO}_{2}}$. In other terms, the final unconditional means include the contributions of different conditional flamelets (after $\beta$-PDF integration) at the same level of progress of reaction described by the choice of $Y_{c}$. The physical interpretation of a presumed-PDF model based on a normalised progress variable would of course be quite different, since in that case the "same level of progress of reaction" would correspond to the same adimensional distance between the inert mixture and the steady flamelet solution (and therefore possibly quite different values for the non-normalised $\left.\left\langle Y_{c} \mid \chi_{\mathrm{st}}\right\rangle\right)$.

\subsection{Final parametrisation}

We store the unconditional mean properties as functions of $\widetilde{Z}, \widetilde{Z^{\prime \prime 2}}, \widetilde{\chi_{\text {st }}}$ and $\widetilde{Y}_{c}$. We have a straightforward relation between $\widetilde{\chi}$ and $\widetilde{\chi_{\mathrm{st}}}$ :

$$
\widetilde{\chi}\left(\widetilde{Z}, \widetilde{Z^{\prime \prime 2}}, \widetilde{\chi}_{\mathrm{st}}\right)=\widetilde{\chi}_{\mathrm{st}} \widetilde{\mathcal{F}_{\mathrm{st}}^{\infty}}\left(\widetilde{Z}, \widetilde{Z^{\prime \prime 2}}\right),
$$

where the mean value $\widetilde{\mathcal{F}_{\text {st }}^{\infty}}$ is obtained from the $\beta$-PDF integration of (13) in $z$-space, and is stored in the lookup table. The replacement of $\left\langle Y_{c} \mid \chi_{\text {st }}\right\rangle$ by $\widetilde{Y}_{c}$ is done through a re-interpolation of the final table, since (25) and (26) are also applied to $\hat{y_{c}}$.

\section{Turbulence model and progress variable equation}

\subsection{Second-moment closure}

We solve the RANS equations using the LRR-IPM secondmoment closure [50] using the formulation described in [51] for the modelling of the scalar fluxes. Standard equations are solved for mean continuity, mean momentum, Reynolds stresses $\widetilde{u_{i}^{\prime \prime} u_{j}^{\prime \prime}}$, turbulent dissipation $\epsilon$, mean mixture fraction $\widetilde{Z}$, its variance $\widetilde{Z^{\prime \prime 2}}$ and the scalar fluxes $\widetilde{u_{i}^{\prime \prime} Z^{\prime \prime}}$, as detailed in [51]. We recall here the transport equations for the mean mixture fraction and its variance, since they directly enter the turbulent combustion model. Under the assumption of high Reynolds number, the mean laminar diffusion terms are neglected:

$$
\begin{aligned}
& \frac{\partial \bar{\rho} \widetilde{Z}}{\partial t}+\frac{\partial \bar{\rho} \widetilde{U_{j}} \widetilde{Z}}{\partial x_{j}}=-\frac{\partial \bar{\rho} \widetilde{u_{j}^{\prime \prime} Z^{\prime \prime}}}{\partial x_{j}}, \\
& \frac{\partial \bar{\rho} \widetilde{Z^{\prime \prime 2}}}{\partial t}+\frac{\partial \bar{\rho} \widetilde{U_{j}} \widetilde{Z^{\prime \prime 2}}}{\partial x_{j}}+2 \widetilde{\rho} \widetilde{u_{j}^{\prime \prime} Z^{\prime \prime}} \frac{\partial \widetilde{Z}}{\partial x_{j}}=-\frac{\partial \bar{\rho} \widetilde{u_{j}^{\prime \prime} Z^{\prime \prime 2}}}{\partial x_{j}}-\bar{\rho} \widetilde{\chi} .
\end{aligned}
$$

On the right hand side of the modelled variance transport equation, the triple correlation term is modelled using the DalyHarlow generalised gradient diffusion model, and the mean scalar dissipation rate is modelled in a standard way as:

$$
\widetilde{\chi}=C_{\phi} \omega \widetilde{Z^{\prime \prime 2}} \text { with } \omega=\epsilon / k,
$$

where $\omega$ is the turbulent frequency and $k=\widetilde{u_{i}^{\prime \prime} u_{i}^{\prime \prime}} / 2$ is the turbulent kinetic energy. The choice of the value of the constant $C_{\phi}$ will be discussed in Section 6.1. We recall that together with this model for $\tilde{\chi}$, the shape of the scalar dissipation rate PDF is also determined by the choice of the value of the fixed variance $\sigma^{2}$ introduced in Section 3.5. The influence of this parameter will also be discussed in the following.

Two constant values are adjusted: in the turbulent dissipation equation the usual modification for round jets is applied by setting $C_{\epsilon 1}=1.6$, and in the scalar flux model the standard value of the Monin's constant $C_{\phi 1}=3$ is increased to $C_{\phi 1}=5$. The latter leads to better agreement of mean mixture fraction with experimental results. A similar modification was applied in [15], where a gradient diffusion closure was used, by increasing the turbulent Schmidt number value. 


\subsection{Mean progress variable}

In order to have a complete model, we need to solve modelled equations for the mean progress variable $\widetilde{Y}_{c}$ and its scalar fluxes $\widetilde{u_{i}^{\prime \prime} Y_{c}^{\prime \prime}}$. A first model can be derived in the same way as the other RANS equations: starting from the exact NavierStokes transport equations and from the exact scalar transport equations and averaging (and neglecting the mean laminar diffusion terms under the assumption of high-Reynolds number flow). In this case, by combining the transport equations of the three mass fractions $Y_{\mathrm{H}_{2} \mathrm{O}}, Y_{\mathrm{HO}_{2}}$ and $Y_{\mathrm{H}_{2}}$ according to (5) we obtain:

$$
\frac{\partial \bar{\rho} \widetilde{Y}_{c}}{\partial t}+\frac{\partial \bar{\rho} \widetilde{U}_{j} \widetilde{Y}_{c}}{\partial x_{j}}=-\frac{\partial \bar{\rho} \widetilde{u_{j}^{\prime \prime} Y_{c}^{\prime \prime}}}{\partial x_{j}}+\bar{\rho} \widetilde{\dot{\omega}_{c}}
$$

where for the terms on the right hand side the same scalar-flux model as for mixture fraction can be used and where the mean reaction term $\tilde{\dot{\omega}}_{c}$ can be retrieved from the pre-integrated unsteady flamelet manifold.

Exact species equations with UFPV assumption. However, the fundamental assumption of the turbulent combustion model is to consider that the underlying structure of the turbulent flame corresponds to unsteady flamelets. In this sense, the exact equations for species mass fractions under the unsteady flamelet model assumption is given by Equation (11) (with given fixedin-time $\chi$-profile), and not by the exact scalar equations used to derive (31). In other terms, we should consider the Lagrangian flamelet equations as a starting point, as introduced in [3]. As shown in the a priori study at the end of Section 2 , instead of considering all species mass fraction, we could simply solve (14) for the progress variable on the Lagrangian flamelet lines. As shown by Pitsch and Steiner [3], invoking the flamelet assumption of thin reaction zones, the corresponding exact flamelet equation in a Eulerian system reads:

$$
\frac{\partial \rho Y_{c}}{\partial t}+\frac{\partial \rho U_{j} Y_{c}}{\partial x_{j}}=\rho \dot{Y}_{c},
$$

where we introduced the notation $\dot{Y}_{c}$ from (14). We can now propose the following modelled equation for $\widetilde{Y}_{c}$ :

$$
\frac{\partial \bar{\rho} \widetilde{Y}_{c}}{\partial t}+\frac{\partial \bar{\rho} \widetilde{U}_{j} \widetilde{Y}_{c}}{\partial x_{j}}=-\frac{\partial \bar{\rho} \widetilde{u_{j}^{\prime \prime} Y_{c}^{\prime \prime}}}{\partial x_{j}}+\bar{\rho} \tilde{\dot{Y}}_{c}
$$

where the last term can be retrieved from the pre-integrated UFPV manifold. This is the kind of modelled equation used by Michel et al. [12] for the mean progress variable when using approximated diffusion flamelets.

Figure 9 illustrates how different the source terms $\dot{\omega}_{c}$ and $\dot{Y}_{c}$ are around stoichiometry. The source term $\dot{Y}_{c}$ goes to zero on the steady flamelet lines and is negative below the unstable branch, while $\dot{\omega}_{c}$ is positive everywhere. As recalled in the introduction, an equation including $\dot{\omega}_{c}$ is usually used in steady flamelet approaches - formally the same as (31). However, in such SFPV models the progress variable $\mathcal{Y}_{c}$ corresponds to the 'steady flame combustion product mass fraction' and is a
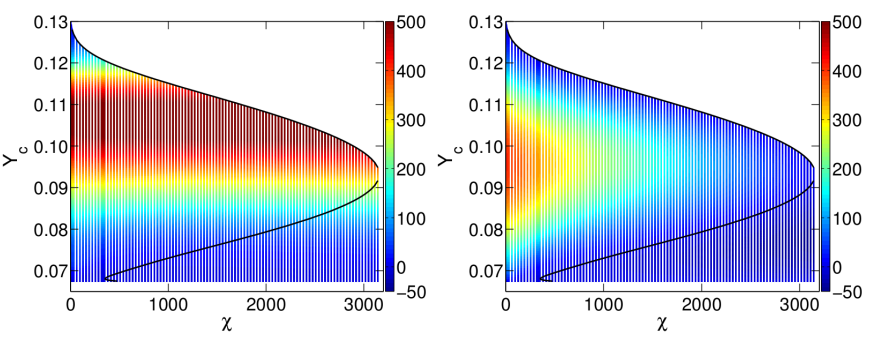

Figure 9: Contours of the source terms $\dot{\omega}_{c}$ (left) and $\dot{Y}_{c}$ (right) at stoichiometric mixture fraction, represented in $\left(\chi, Y_{c}\right)$-space.

way to parametrise the steady flamelet properties in composition space instead of considering the scalar dissipation rate $\chi$. In this case, the mean transport equation of the form of (31) is somehow arbitrary and its resolution (including clipping if necessary) together with the presumed PDF model implies a shape for the PDF of $\chi$.

In the case of unsteady flamelets, the progress variable $Y_{c}$ is of different nature of $\boldsymbol{y}_{c}$ and both equations (31) and (33) can be given a physical interpretation. We discussed how (33) is consistent with the basic UFPV model assumptions exposed in Section 2.

\section{Test case and numerical set-up}

The numerical set-up is very similar to the carefully chosen one considered by Cao et al. [21], also in a RANS framework (with transported PDF modelling in that case). Starting at the fuel injector exit, the computational domain - 50D-long (in axial direction) and $20 D$-wide (in radial direction), with $D=4.57 \mathrm{~mm}$ the injector diameter - is a half-plane of the $2 \mathrm{D}$ axisymmetric geometry considered. A $108 \times 108$ cell nonuniform grid is used, stretched in both axial and radial directions (with 13 uniform grid cells in radial direction above the fuel injector: between $r=0$ and $r=R=D / 2$ ).

Symmetry conditions are imposed on the symmerty axis, a free-slip boundary is specified on the outer boundary and a convective outlet condition is used. The inlet profiles are specified in such a way that the fluctuating axial and radial velocity profiles, $u^{\prime}$ and $v^{\prime}$, are similar to the experimental profiles used in [21], where the influence of the inlet boundary conditions was carefully studied. In this RANS framework, the influence of the wall flows on the inner and outer sides of the fuel nozzle are simply accounted for at the injector exit by specifying the turbulent profiles for mean velocity components, Reynolds stresses and turbulent dissipation. The axial mean velocity profile at the injector exit is specified as $\widetilde{U}=U_{\text {norm }}(1.01-r / R)^{1 / 6}$, the coflow mean velocity $\widetilde{U}=3.5 \mathrm{~m} / \mathrm{s}$ is imposed at $r>1.88 R$. For $R<r<1.88 R$, both profiles are connected with an exponential decay $\widetilde{U}=\alpha+\beta$. $\exp \left(-\gamma r^{2}\right)$, with $\gamma \approx 480000$, leading to a similar profile as used in [21]. This axial mean velocity profile is normalised with $U_{\text {norm }}$ such that the correct experimental bulk velocity of $107 \mathrm{~m} / \mathrm{s}$ is imposed at the injector exit (i.e. correct fuel mass flow rate). The radial mean velocity is set to zero. The Reynolds stress profiles are specified as $\widetilde{u^{\prime \prime 2}}=u^{\prime} u^{\prime}$ (axial), 
$\widetilde{v^{\prime \prime 2}}=v^{\prime} v^{\prime}$ (radial) and $\widetilde{w^{\prime \prime 2}}=\widetilde{v^{\prime \prime 2}}$ (azimuthal). The turbulent shear stress is specified in a similar way as in [21] such that $\widetilde{u^{\prime \prime} v^{\prime \prime}}=\rho_{u v} u^{\prime} v^{\prime}$, with $\rho_{u v}=0.4(r / R)$ for $r \leq R$ and $\rho_{u v}=0.4$ for $r>R$. Finally, the turbulent dissipation profile is specified by supposing that the dissipation term is equal to the production term in the turbulent kinetic energy transport equation: $\epsilon=-\widetilde{u^{\prime \prime} v^{\prime \prime}} \cdot \partial \widetilde{U} / \partial r$.

As already mentioned, the Reynolds-stress and Reynoldsflux models corresponding to the variable $C_{0}$ formulation of the Generalised Langevin Model presented in [51] are used (here in correspondence with the LRR-IPM Reynolds stress model), with two modified constants: $C_{\epsilon 1}=1.6$ in the turbulent dissipation equation and $C_{\phi 1}=5$ in the scalar-flux equations. The $2 \mathrm{D}$ axisymmetric calculations are performed with the same computer code PDFD as in [51].

The lookup table is discretised in 100 uniform intervals in $\widetilde{Z}$ between 0 and 1, 20 uniform intervals in $S=\widetilde{Z^{\prime \prime 2}} /[\widetilde{Z}(1-\widetilde{Z})]$ between 0 and 0.2 and 50 uniform intervals in $\widetilde{Y}_{c}$ between $\widetilde{Y_{c}^{\min }}\left(\widetilde{Z}, \widetilde{Z^{\prime \prime 2}}\right)$ and $\widetilde{Y_{c}^{\max }}\left(\widetilde{Z}, \widetilde{Z^{\prime \prime 2}}\right)$. A logarithmic grid is used to store the lookup table in $\widetilde{\chi_{\mathrm{st}}}$, using 41 uniform intervals between $\ln \left(\chi_{\text {st,MIN }}\right)$ and $\ln \left(2 \chi_{\text {st,MAX }}\right)$ (corresponding to approximately 10 intervals per order of magnitude).

\section{Results}

Different calculations are made both with $\dot{\omega}_{c}$ and $\dot{Y}_{c}$ as reaction term in the modelled transport equation for the mean progress variable, with different values of $C_{\phi}$ and $\sigma$ and for different coflow temperatures. When using $\dot{Y}_{c}$ we take as reference calculation the one with coflow temperature $T_{\text {cof }}=1062 \mathrm{~K}$, and when using $\dot{\omega}_{c}$ the one with $T_{\text {cof }}=1053 \mathrm{~K}$. In both cases we take $C_{\phi}=3$ and $\sigma=1$ as reference values.

The reason for the $9 \mathrm{~K}$ difference in coflow temperature in order to match the correct lift-off height, whether $\dot{Y}_{c}$ or $\dot{\omega}_{c}$ is used, will be seen in Figure 18. At these temperatures, the modelled flames are stabilised around stoichiometry: the use of $\dot{Y}_{c}$, including diffusion effects, leads to a "less reactive flame" than when using $\dot{\omega}_{c}$ (as can be seen in Figure 9) and requires a higher coflow temperature in order to lead to the same lift-off height.

\subsection{Influence of $C_{\phi}$ and main model properties}

We consider the calculation at $T_{\text {cof }}=1062 \mathrm{~K}$ using $\dot{Y}_{c}$ and $\sigma=1$ (the same observations are made for other cases). Figure 10 shows the influence of $C_{\phi}$ on the results. First of all, we stress the fact that very good results are obtained for mean mixture fraction (comparable to the best results of the literature like $[21,25])$ due to the usual adjustment of the constant $C_{\epsilon 1}=1.6$ for round jets, and in particular due to the adjustment of the constant $C_{\phi 1}=5$ in the scalar-flux model. We observe the impact of the choice of the value of $C_{\phi}$ on the results for mixture fraction variance, where the value $C_{\phi}=3$ gives the best agreement with the experimental data. Note that, as in [15] or [25], the small level of fluctuations in the coflow is not captured, while it was in the transported PDF results of [21].

In Figure 11, we plot the value of the mean scalar dissipation rate following the mean stoichiometric isoline as a function of

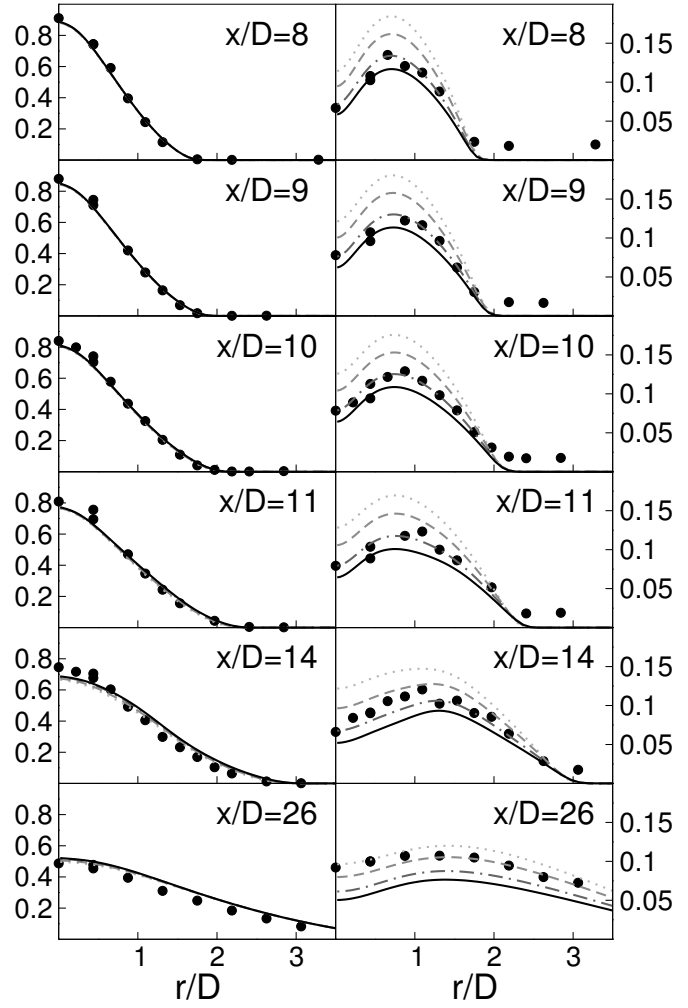

Figure 10: Radial profiles of mean mixture fraction (left) and its rms fluctuation (right). Symbols: experimental data / Lines: results using $\dot{Y}_{c}$ and $T_{\text {cof }}=1062 \mathrm{~K}$ (with $\sigma=1$ ). Dotted lines: $C_{\phi}=1.5$. Dashed lines: $C_{\phi}=2$. Dashed-dotted lines: $C_{\phi}=3$. Solid lines: $C_{\phi}=4$.

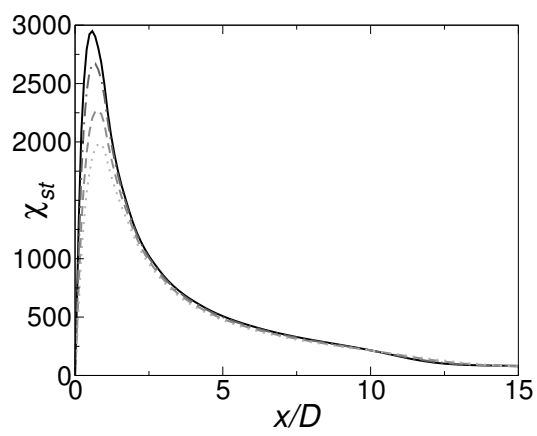

Figure 11: Mean scalar dissipation rate following the mean stoiciometric isoline in the calculation using $\dot{Y}_{c}$ and $T_{\text {cof }}=1062 \mathrm{~K}$ (with $\sigma=1$ ), for different values of $C_{\phi}$. Legend: see Fig. 10.

the axial distance. We observe, that the value of $C_{\phi}$ does not have almost any influence on the mean scalar dissipation rate anywhere in the flow (except very close to the injector exit). This is simply explained by looking at equation (30) where two effects counterbalance, since increasing $C_{\phi}$ implies lower values of $\widetilde{Z^{\prime \prime 2}}$, as observed in Figure 10. This low dependency of $\widetilde{\chi}$ is in line with the observed low dependency of the results on the value of $C_{\phi}$ in previous transported PDF modelling for different mixing models [21].

Figures 12 and 13 show the radial profiles of mean temperature and mean mass fraction of $\mathrm{H}_{2} \mathrm{O}$ and their fluctuations. The results for these properties are similar and provide a good esti- 


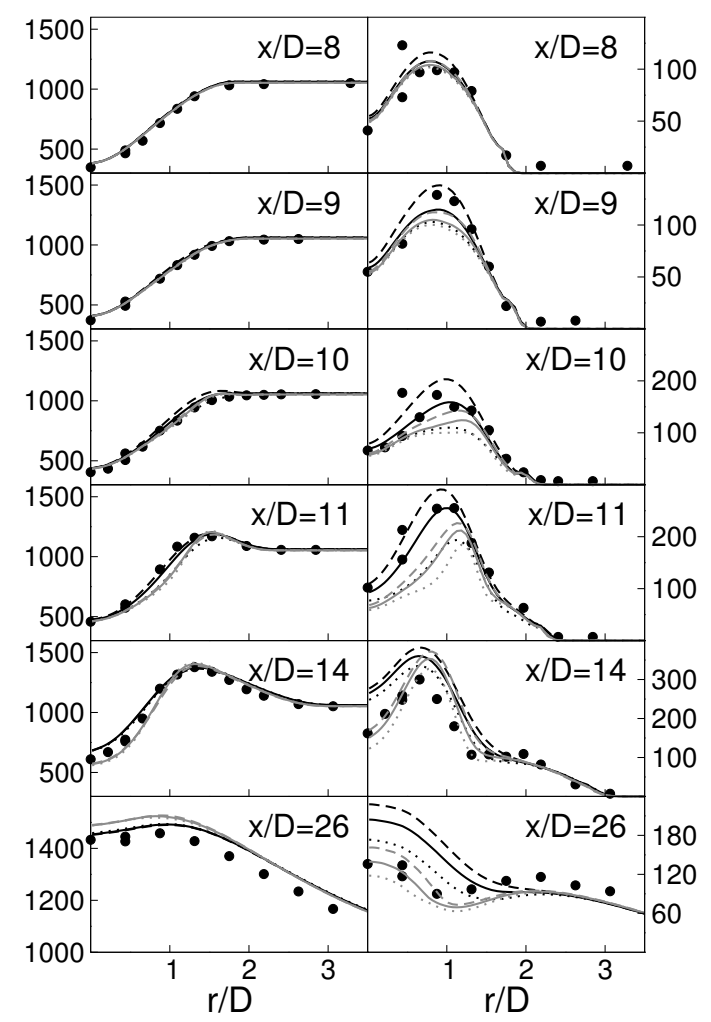

Figure 12: Radial profiles of mean temperature (left) and its rms fluctuation (right). Symbols: experimental data. Black lines: $\dot{Y}_{c}$ and $T_{\text {cof }}=1062 \mathrm{~K}$ (with $C_{\phi}=3$ ). Grey lines: $\dot{\omega}_{c}$ and $T_{\text {cof }}=1053 \mathrm{~K}$ (with $C_{\phi}=3$ ). Dotted lines: $\delta$-PDF for $\chi_{\mathrm{st}}$. Solid lines: $\sigma=1$. Dashed lines: $\sigma=\sqrt{2}$.

mate of the quality of the prediction of the progress variable $Y_{c}$. We observe that the mean values are well predicted for the two cases considered $\left(\dot{Y}_{c}\right.$ and $T_{\text {cof }}=1062 \mathrm{~K}$, and $\dot{\omega}_{c}$ and $\left.T_{\text {cof }}=1053 \mathrm{~K}\right)$, again with the same level of agreement as the best transported PDF results [21, 25], and better than the LESCMC results of [33].

At this point, we could verify that we obtain results in good correspondence with experimental data for three of the four properties on which our turbulent combustion model is based: $\widetilde{Z}, \widetilde{Z^{\prime \prime 2}}$ and $\widetilde{Y}_{c}$. These results are obtained with $C_{\phi}=3$, both using $\dot{\omega}_{c}$ or $\dot{Y}_{c}$ as reaction term in the mean progress variable transport equation, by adjusting the coflow temperature. Concerning $\widetilde{\chi}$, no data is available and we simply stick to the standard model (30). We can now consider the predictive capabilities of the proposed turbulent combustion model for the other properties and their fluctuations. As described in Section 3, the results for all the other properties are a direct consequence of the UFPV assumption, together with the model for presumed shape of the joint PDF. In particular, the fluctuations of the progress variable and of the tabulated properties are directly related to the model for the fluctuations of $Z$ and $\chi$.

\subsection{Influence of $\sigma$}

The columns on the right hand side of Figures 12 and 13 give an idea of the fluctuations of the progress variable. The variances are obtained from the tabulated mean properties and

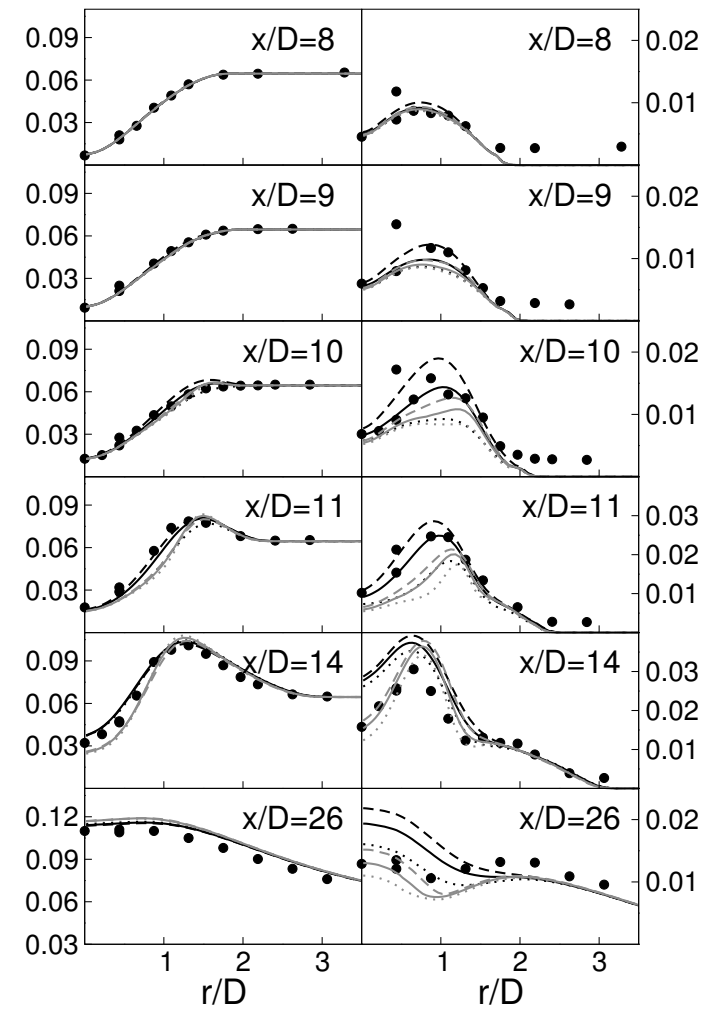

Figure 13: Radial profiles of mean mass fraction of $\mathrm{H}_{2} \mathrm{O}$ (left) and its rms fluctuation (right). Legend: see Fig. 12.

mean of the square, for instance for the temperature: $\widetilde{T^{\prime \prime 2}}=$ $\widetilde{T^{2}}-\widetilde{T}^{2}$. Close to the injector exit, both the centreline value and the peak value of the radial profiles are correctly captured when using $\dot{Y}_{c}$, while further downstream, they are correctly captured using $\dot{\omega}_{c}$. We observe that the fluctuation predictions are sensitive to the prescribed fixed variance $\sigma^{2}$ in the PDF of the logarithm of $\chi_{\text {st }}$ (especially when using $\dot{Y}_{c}$ ), whereas mean values are not sensitive to this model parameter.

The fuel and oxidiser mass fraction results are shown in Figures 14 and 15. Good agreement with experimental data is obtained for the mean radial profiles. Concerning the fluctuations, similar observations can be made as for temperature. However, for these reactants which are consumed in the reaction zone, we observe less sensitivity to $\sigma$, or to the use of $\dot{Y}_{c}$ or $\dot{\omega}_{c}$ as compared to the main combustion product and temperature.

As expected, the most sensitive property is the mass fraction of $\mathrm{OH}$ radical as shown in Figure 16. The differences are especially important at the flame base, and the results become similar further downstream. In this case, the differences are quite large whether $\dot{Y}_{c}$ or $\dot{\omega}_{c}$ is used as reaction term. The best agreement with experimental data is obtained with $\dot{Y}_{c}$, both for the mean and the fluctuations, similar to the best results of the literature as for instance in $[21,25,33]$. The choice of $\sigma$ also has a strong impact on the results, even for the mean mass fraction. Again, the values $\sigma^{2}=1$ and $\sigma^{2}=2$ seem reasonable, with the best results obtained with $\sigma^{2}=2$ just before the flame base. However, the $\delta$-PDF assumption for $\chi_{\text {st }}$ leads to poor results for the fluctuations before the flame base. 


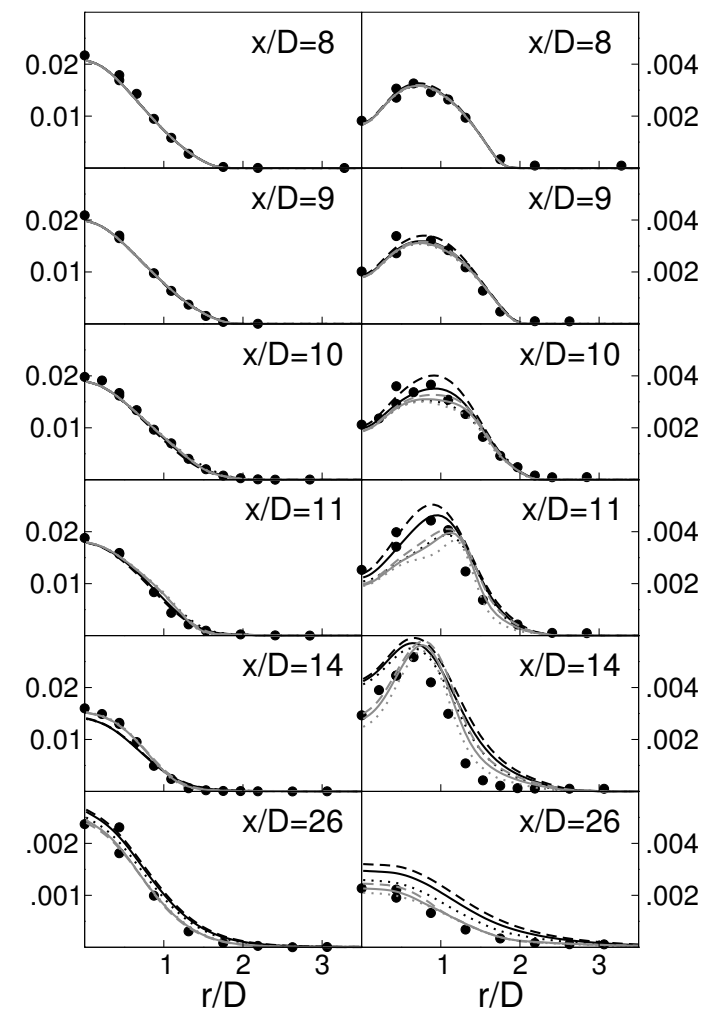

Figure 14: Radial profiles of mean mass fraction of $\mathrm{H}_{2}$ (left) and its rms fluctuation (right). Legend: see Fig. 12.

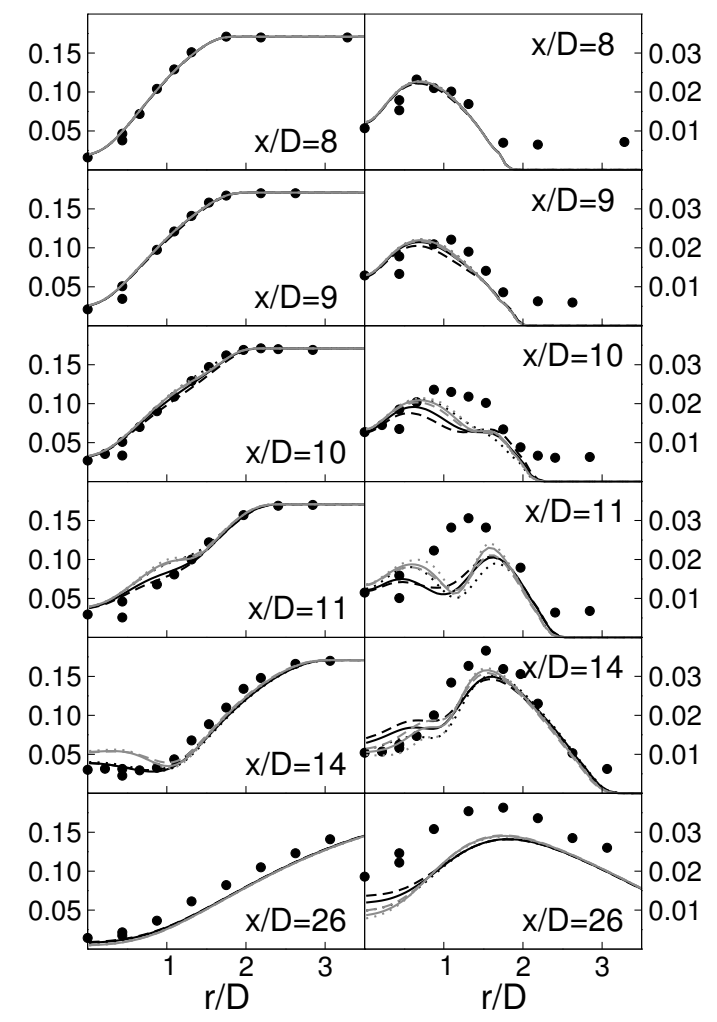

Figure 15: Radial profiles of mean mass fraction of $\mathrm{O}_{2}$ (left) and its rms fluctuation (right). Legend: see Fig. 12.

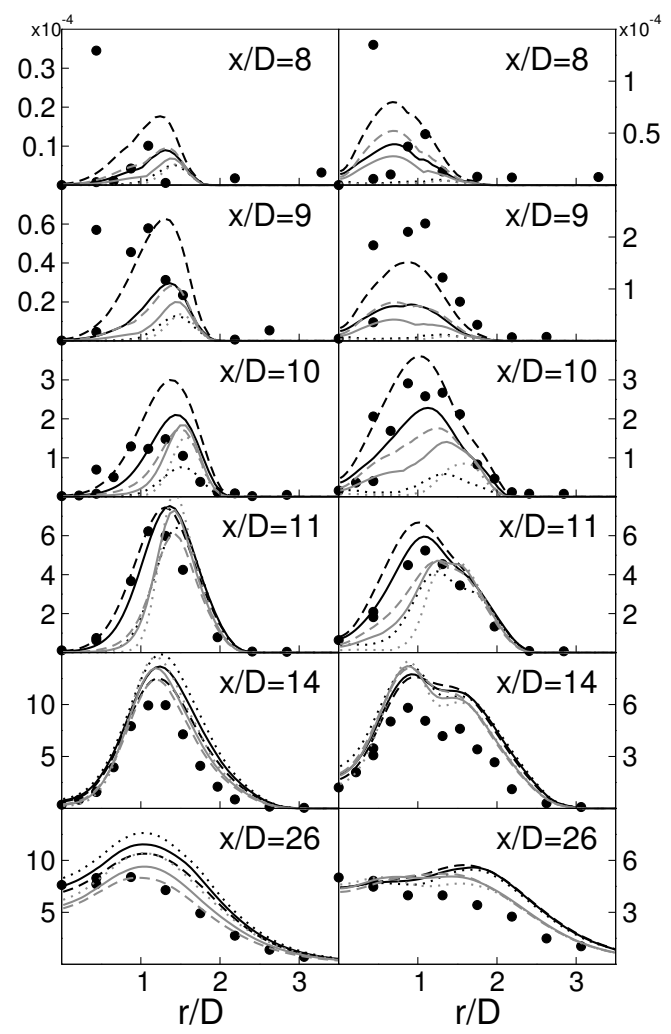

Figure 16: Radial profiles of mean mass fraction of $\mathrm{OH}$ (left) and its rms fluctuation (right). Legend: see Fig. 12.

\subsection{Influence of coflow temperature, use of $\dot{Y}_{c}$ or $\dot{\omega}_{c}$}
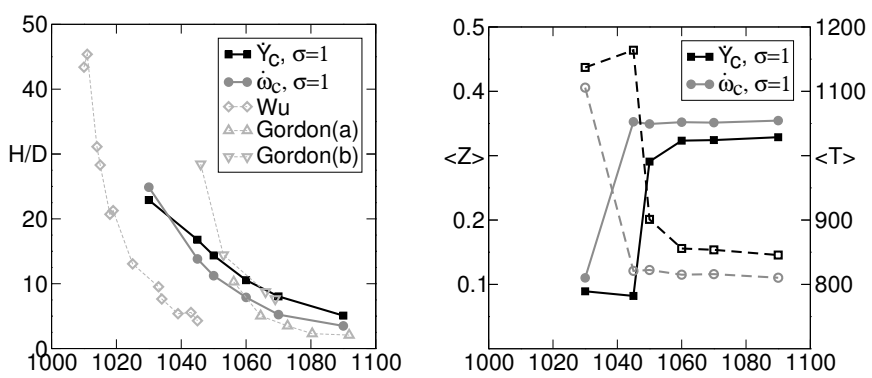

Figure 17: Left plot: flame lift-off height as function of coflow temperature (the light grey lines with diamonds and triangles represent experimental data). Right plot: Corresponding mean mixture fraction (dashed lines) and mean temperature (solid lines) at the flame base.

Figure 17 shows the dependence of flame lift-off height $H$ on the coflow temperature $T_{\text {cof }}$. As in [21], the distance $H$ corresponding to the base of the modelled lifted flame is evaluated as the axial distance where $Y_{\mathrm{OH}}=2 \cdot 10^{-4}$. Results are shown both using $\dot{Y}_{c}$ or $\dot{\omega}_{c}$ as reaction term, with $\sigma=1$ (similar trends are obtained with the $\delta$-PDF assumption for $\chi_{\text {st }}$ or with $\sigma=\sqrt{2}$ ). The differences between the experimental data sets illustrate the high sensitivity of this flame to the coflow temperature. This justifies the adjustment of the coflow temperature in the modelling in order to match a given lift-off height. On the other hand, the significant information given by these data sets is the trend of the dependence of $H$ on $T_{\text {cof }}$. The model should be able 
to reproduce this dependence. The trend of both curves (using $\dot{Y}_{c}$ or $\dot{\omega}_{c}$ ) is in correspondence with the experimental data for high coflow temperature. However, at low coflow temperatures the calculations do not predict an increase in lift-off height as high as expected.

The plot on the right hand side of Figure 17 shows that at high coflow temperatures, the flame base corresponds to lean mixture fraction values typical of autoignition. However, we observe that at lower coflow temperatures, the flame base corresponds to mixtures close to stoichiometry and to temperatures which are in principle too low for autoignition to occur: i.e. below the typical crossover temperature for hydrogen chemistry at atmospheric pressures of the order of $T_{c} \approx 950 \mathrm{~K}$ (the recent review of Sánchez and Williams [52] on hydrogen chemistry provides details about the crossover temperature $T_{c}$, where the rates of branching and recombination reactions are equal). Therefore, it appears that at lower coflow temperature our model does not predict autoignition at the base of the flame.

The similar model presented in [15] using $\dot{\omega}_{c}$ leads to results very similar to the one shown on the left hand side of Figure 17, but no further study of flame stabilisation is provided. However, in the RANS-transported PDF of [22] or in the LESCMC of [33], the budget analysis leads to the conclusion that autoignition is the main stabilisation mechanism for all coflow temperatures considered.

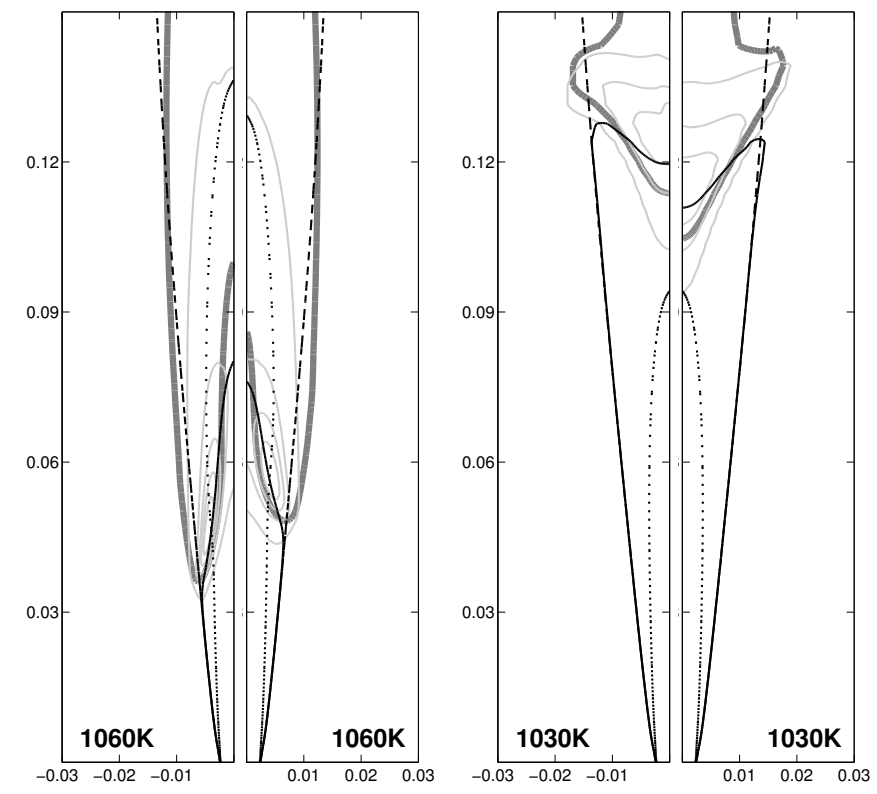

Figure 18: Contours for the lifted flame at two different coflow temperatures. Left: using $\dot{\omega}_{c} /$ Right: using $\dot{Y}_{c}$. Solid black line: mean temperature isoline at $\widetilde{T}=950 \mathrm{~K}$. Dashed black line: mean temperature isoline at $\widetilde{T}=950 \mathrm{~K}$ in the inert flow. Dotted line: stoichiometric isoline. Light grey lines: isocontours of mean reaction term $\left(\widetilde{\dot{\omega}_{c}}\right.$ on the left and $\widetilde{\dot{Y}}_{c}$ on the right). Thick dark grey lines: mean $\mathrm{OH}$ mass fraction isoline at $\widetilde{Y}_{\mathrm{OH}}=2 \cdot 10^{-4}$.

Figure 18 shows two qualitatively different flames obtained with our model at low or high coflow temperature (as shown in the figure, the same trend is observed when using $\dot{\omega}_{c}$ or $\dot{Y}_{c}$ ). At $T_{\text {cof }}=1060 \mathrm{~K}$, we obtain a lifted flame stabilised at lean mean mixture fraction values around the mean temperature iso- line $\widetilde{T}=T_{c}=950 \mathrm{~K}$. In this case, we see that the reaction zone covers both lean and rich regions and that the high reaction rate values are around stoichiometry.

At $T_{\text {cof }}=1030 \mathrm{~K}$ a very different mean flame is obtained. A $\mathrm{V}$-shape is observed instead of the W-shape observed at high coflow temperature. Note that this steady flame is obtained by starting the calculation from an inert solution at $T_{\text {cof }}=1030 \mathrm{~K}$ (obtained by switching off the reaction term in the transport equation for $\widetilde{Y}_{c}$ ). In this case, due to longer ignition delay times, the reaction zone is now in a lean mean mixture, in a downstream region with lower mean axial velocity. Those two aspects are likely to be the most relevant to explain why our model leads to a location of the flame base on the centreline at a mean temperature lower than $T_{c}=950 \mathrm{~K}$. The resulting Vshape of the modelled mean flame can explain why the lift-off height is underestimated.

The reason for such a behaviour of the model at low coflow temperature still needs to be better understood. However, these configurations correspond to very sensitive conditions (which are avoided in practical applications), due to the fact that the coflow temperatures are close to the crossover temperature $T_{c}$.

\section{Conclusions}

A complete turbulent combustion model based on igniting and extinguishing laminar diffusion flamelets (at unity Lewis number) has been presented and discussed.

Different time evolutions of igniting and extinguishing unsteady flamelets are observed depending on whether they are resolved in physical space or in mixture fraction space: while the scalar dissipation rate is fixed in the latter case, it strongly varies in time when resolving the problem in physical space. A first qualitative comparison tends to show that these different time evolutions do not lead to large differences between the manifolds in $\left(Z, \chi, Y_{c}\right)$-space.

In the considered UFPV approach proposed for autoignition problems, the progress variable is defined including the main combustion product $\left(\mathrm{H}_{2} \mathrm{O}\right)$ and a key radical species for autoignition $\left(\mathrm{HO}_{2}\right)$. The transport equation to be used for this progress variable is discussed. It appears that, in principle, a transport equation consistent with the modelling assumptions should not include the chemical reaction term $\dot{\omega}_{c}$ as usually done in steady flamelet approaches, but rather a source term $\dot{Y}_{c}$ including both chemical reaction and molecular diffusion in mixture fraction space, as derived from the Lagrangian flamelet equations.

The presumed-PDF model is presented in a detailed way and leads to a formulation of the problem in a RANS framework in terms of mean mixture fraction $\widetilde{Z}$, its variance $\widetilde{Z^{\prime \prime 2}}$ and mean scalar dissipation rate $\widetilde{\chi}$. The novelty of the model is that we assume that there are no fluctuations of the unsteady flamelet Lagrangian time at given strain rate. This $\delta$-distribution for the conditional PDF allows to reparametrise the final table in terms of the mean progress variable. The dependence of the progress variable on mixture fraction is included, corresponding to the time evolution of igniting flamelets at given strain rates. The 
strain rate fluctuations are included for identical values of the non-normalised progress variable. This formulation implies a model for the fluctuations of the progress variable and of the other flamelet properties which only depend on the fluctuations in $Z$ due to the $\beta$-PDF and the fluctuations in $\chi_{\text {st }}$ due to the lognormal distribution with fixed variance $\sigma$. In this framework, we may consider in a straightforward way in the future the use of different presumed PDF shapes for $Z$ or $\chi_{\mathrm{st}}$, in particular if the model would be extended to LES.

The model is applied to a $\mathrm{H}_{2} / \mathrm{N}_{2}$ turbulent lifted flame, and leads to very good results compared to the experimental data for the flame stabilised around ten injector diameters, after adjusting the coflow temperature. Both formulations using $\dot{\omega}_{c}$ and $\dot{Y}_{c}$ lead to good agreement with experimental data (with different adjustments of the coflow temperature). The very good prediction of mean mixture fraction is obtained by adjusting the constant $C_{\phi 1}=5$ in the second-moment closure model for the scalar-fluxes, and good results for mixture fraction variance are obtained by setting the constant $C_{\phi}=3$ in the model for mean scalar dissipation rate. We show that the results for fluctuations of temperature and $\mathrm{H}_{2} \mathrm{O}$ mass fraction are quite sensitive to the choice of $\sigma$, and that both the mean and the fluctuations of $\mathrm{OH}$ mass fraction are sensitive to this parameter.

Finally, we show that our model predicts the correct trend for the dependence of flame lift-off height at high coflow temperatures. However, at low coflow temperature, the model leads to an underestimation of the flame lift-off height, corresponding to $\mathrm{V}$-shape flames stabilised on the centerline instead of the W-shape flames obtained at higher coflow temperatures. It still needs to be understood why such effects are predicted by the model, keeping in mind that these limit conditions (very close to the crossover temperature) are not desirable in practical devices.

\section{References}

[1] N. Peters. Combust. Sci. Technol., 30 (1983) 1-17.

[2] N. Peters. Turbulent Combustion, Cambridge University Press, Cambridge, 2000.

[3] H. Pitsch and H. Steiner. Physics of Fluids, 12 (2000) 2541-2554.

[4] H. Pitsch, and M. Ihme. 43rd AIAA Aerospace Sciences Meeting, Paper 2004-557.

[5] M. Ihme and H. Pitsch. Combust. Flame, 155 (1-2) (2008) 70-89.

[6] M. Ihme and Y.C. See. Combust. Flame, 157 (2010) 1850-1862.

[7] S.K. Sadasivuni. LES Modelling of Non-premixed and Partially Premixed Turbulent Flames, Ph.D. thesis, Loughborough University (2009). https://dspace.lboro.ac.uk/2134/5804

[8] S.K. Sadasivuni, W. Malalasekera and S.S. Ibrahim Russian Journal of Physical Chemistry B, 4 (2010) 465-474.

[9] I. Dhuchakallaya, P. Rattanadecho and P. Watkins. Applied Thermal Engineering, 52 (2013) 420-427.

[10] C. Bajaj, M. Ameen and J. Abraham Combustion Science and Technology, 185 (2013), 454-472.

[11] J.-B. Michel, O. Colin and D. Veynante. Flow, Turb. Combust., 83 (2009) 33-60.

[12] J.-B. Michel, O. Colin, C. Angelberger and D. Veynante. Combust. Flame, 156 (2009) 1318-1331.

[13] J.-B. Michel, O. Colin and C. Angelberger. Combust. Flame, 157 (2010) 701-714.

[14] P.-D. Nguyen, L. Vervisch, V. Subramanian and P. Domingo. Combust. Flame, 157 (2010) 43-61.
[15] R. Vicquelin, Tabulation de la cinétique chimique pour la modélisation et la simulation de la combustion turbulente, Ph.D. thesis, Ecole Centrale Paris (2010).

[16] R. Cabra, T. Myhrvold, J.Y. Chen, R.W. Dibble, A.N. Karpetis and R.S. Barlow. Proc. Combust. Inst., 29 (2002) 1881-1888.

[17] http://www.me.berkeley.edu/cal/vcb/data/VCHNData.html

[18] R.L. Gordon, S.H. Starner, A.R. Masri and R.W. Bilger. 5th Asia-Pacific Conference on Combustion, (2005).

[19] Z. Wu, A.R. Masri and R. Bilger. Flow, Turb. Combust., 76 (2006) 61-81.

[20] A.R. Masri, R. Cao, S.B. Pope and G.M. Goldin. Combust. Theory Modell., 8 (2004) 1-22.

[21] R. Cao, S.B. Pope and A.R. Masri. Combust. Flame, 142 (2005) 438-453.

[22] R.L. Gordon, A.R. Masri, S.B. Pope and G.M. Goldin. Combust. Theory Modell., 11 (2007) 351-376.

[23] H. Wang and S.B. Pope. Combust. Theory Modell., 12 (2008) 857-882.

[24] Z. Ren and S.B. Pope. Proc. Combust. Inst., 32 (2009) 1629-1637.

[25] K. Gkagkas and R.P. Lindstedt. Combust. Theory Modell., 13 (2009) 607 643.

[26] J. Lee and Y. Kim. Int. J. Hydrogen Energy, 37 (2012) 18498-18508.

[27] S.M. Mir Najafizadeh, M.T. Sadeghi, R. Sotudeh-Gharebagh and D. Roekaerts. Combust. Flame, 160 (2013) 2928-2940.

[28] S.M. Mir Najafizadeh, M.T. Sadeghi and R. Sotudeh-Gharebagh. Int. J. Hydrogen Energy, 38 (2013) 2510-2522.

[29] S.S. Patwardhan, S. De, K.N. Lakshmisha and B.N. Raghunandan. Proc. Combust. Inst., 32 (2009) 1705-1712.

[30] W.P. Jones and S. Navarro-Martinez. Combust. Flame, 150 (2007) 170187.

[31] C. Duwig and L. Fuchs. Combust. Sci. Technol., 180 (2008) 452-480.

[32] S. Navarro-Martinez and A. Kronenburg. Flow, Turb. Combust., 87 (2011) 377-406.

[33] I. Stanković and B. Merci. Thermal Science, 17 (2013) 763-772.

[34] S-K. Liew, K.N. Bray and J.B. Moss. Combust. Flame, 56 (1984) 199213.

[35] C.D. Pierce and P. Moin J. Fluid Mech., 504 (2004) 73-97.

[36] J.A. van Oijen and L.P.H. de Goey. Combust. Sci. Technol., 161 (2000) 113-137.

[37] O. Gicquel, N. Darabiha and D. Thevenin. Proc. Combust. Inst., 28 (2000) 1901-1908.

[38] B. Fiorina, R. Baron, O. Gicquel, D. Thevenin, S. Carpentier and N. Darabiha. Combust. Theory Modell., 7 (3) (2003) 449-470.

[39] V. Bykov and U. Maas. Combust. Theory Modell., 11 (6) (2007) 839-862.

[40] H. Pitsch. Annu. Rev. Fluid Mech., 38 (2006) 453-482.

[41] R.W. Bilger, S.H. Starner and R.J. Kee. Combust. Flame, 80 (1990) $135-$ 149.

[42] P. Saxena and F.A. Williams. Combust. Flame, 145 (2006) 316-323.

[43] R.J. Kee, J.A. Miller and G.H. Evans. Proc. Combust. Inst., 22 (1988) $1479-$

[44] A.E. Lutz, R.J. Kee, J.F. Grcar and F.M. Rupley. Sandia National Laboratories Report, 96-8243 (1996).

[45] J.F. Grcar. Sandia National Laboratories Report, SAND91-8230 (1991).

[46] L.R. Petzold. Sandia National Laboratories Report, SAND82-8637 (1982).

[47] O.R. Darbyshire and N. Swaminathan. Combust. Sci. Technol., 184 (2012) 2036-2067.

[48] S. Ruan, N. Swaminathan and O. Darbyshire. Combust. Theory Modell., 18 (2) (2014) 295-329.

[49] R. De Meester, B. Naud and B. Merci. Combust. Flame, 159 (2012) 3353-3357.

[50] B.E. Launder, G.J. Reece and W. Rodi. J. Fluid Mech., 68 (1975) 537566.

[51] B. Naud, B. Merci and D. Roekaerts. Flow, Turb. Combust., 85 (2010) 363-382.

[52] A.L. Sánchez and F.A. Williams. Prog. Energy Combust. Sci., 41 (2014) $1-55$. 